\title{
$B_{s, d}^{0} \rightarrow \ell^{+} \ell^{-}$decays in the aligned two-Higgs-doublet model
}

\section{Xin-Qiang $\mathbf{L i},{ }^{a, b}$ Jie $\mathbf{L} \mathbf{u}^{c}$ and Antonio Pich ${ }^{c}$}

${ }^{a}$ Institute of Particle Physics and Key Laboratory of Quark 8 Lepton Physics (MOE), Central China Normal University,

Wuhan, Hubei 430079, P.R. China

${ }^{b}$ State Key Laboratory of Theoretical Physics, Institute of Theoretical Physics,

Chinese Academy of Sciences,

Beijing 100190, China

${ }^{c}$ IFIC, Universitat de València - CSIC,

Apt. Correus 22085, E-46071 València, Spain

E-mail: xqli@itp.ac.cn, lu.jie@ific.uv.es, pich@ific.uv.es

Abstract: The rare decays $B_{s, d}^{0} \rightarrow \ell^{+} \ell^{-}$are analyzed within the general framework of the aligned two-Higgs doublet model. We present a complete one-loop calculation of the relevant short-distance Wilson coefficients, giving a detailed technical summary of our results and comparing them with previous calculations performed in particular limits or approximations. We investigate the impact of various model parameters on the branching ratios and study the phenomenological constraints imposed by present data.

Keywords: Higgs Physics, Rare Decays, Beyond Standard Model, B-Physics

ARXIV EPRINT: 1404.5865 


\section{Contents}

1 Introduction 1

2 The aligned two-Higgs doublet model 4

2.1 Yukawa sector 5

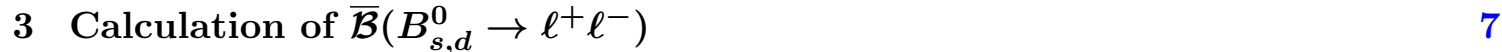

3.1 Effective Hamiltonian $\quad 7$

3.2 Computational method 8

$\begin{array}{lll}3.3 & \text { Wilson coefficients in the SM } & 10\end{array}$

3.4 Wilson coefficients in the A2HDM 13

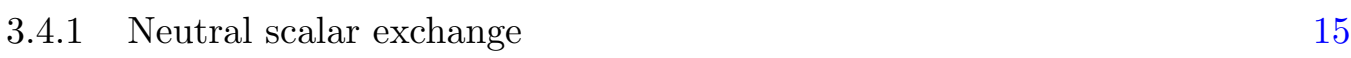

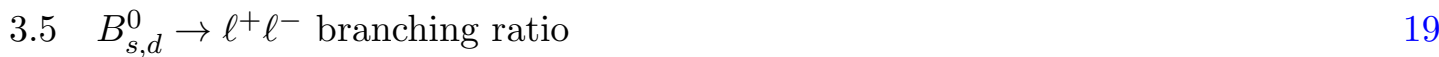

4 Numerical results $\quad 20$

4.1 Input parameters 20

4.2 SM predictions 21

4.3 Results in the A2HDM 22

4.3.1 Choice of model parameters 22

$\begin{array}{lll}4.3 .2 & \text { Small } \varsigma_{d, \ell} & 23\end{array}$

$\begin{array}{lll}4.3 .3 & \text { Large } \varsigma_{d, \ell} & 24\end{array}$

$\begin{array}{lll}4.4 & \mathcal{Z}_{2} \text { symmetric models } & 25\end{array}$

$\begin{array}{llr}5 & \text { Conclusions } & 27\end{array}$

A Scalar-penguin results within the A2HDM 28

\section{Introduction}

The recent discovery of a Higgs-like boson [1,2], with properties compatible with the Standard Model (SM) expectations [3-6], is one of the greatest achievements in the past decades in particle physics and represents a major confirmation of our present theoretical paradigm. The LHC data suggest that the electroweak symmetry breaking (EWSB) is probably realized in the most elegant and simple way, i.e., via the Higgs mechanism implemented through one scalar $\mathrm{SU}(2)_{\mathrm{L}}$ doublet. An obvious question we are now facing is whether the discovered $126 \mathrm{GeV}$ state corresponds to the unique Higgs boson incorporated in the SM, or it is just the first signal of a much richer scenario of EWSB. None of the fundamental principles of the SM forbids the possibility of an enlarged scalar sector associated with the EWSB. 
Among the many possible scenarios for new physics (NP) beyond the SM, the twoHiggs doublet model (2HDM) [7] provides a minimal extension of the scalar sector that naturally accommodates the electroweak (EW) precision tests, giving rise at the same time to a large variety of interesting phenomenological effects $[8,9]$. The scalar spectrum of the model consists of two charged fields, $H^{ \pm}$, and three neutral ones, $h, H$ and $A$, one of which is to be identified with the Higgs-like boson found at the LHC. The direct search for these additional scalar states at high-energy collisions, or through indirect constraints via precision flavour experiments, is an important task for the next years. This will also be helpful to gain further insights into the scalar sector of supersymmetry (SUSY) and other models with similar scalar contents.

Within the SM, flavour-changing neutral current (FCNC) interactions are forbidden at tree level, and highly suppressed at higher orders, due to the Glashow-IliopoulosMaiani (GIM) mechanism [10]. In a generic 2HDM, however, tree-level FCNC interactions generally exist, through non-diagonal couplings of neutral scalars to fermions. The unwanted FCNCs can be eliminated, imposing on the Lagrangian an ad-hoc discrete $\mathcal{Z}_{2}$ symmetry; depending on the different possible $\mathcal{Z}_{2}$ charge assignments, this results in four types of $2 \mathrm{HDMs}$ (I, II, X and Y) [8, 9], all satisfying the hypothesis of natural flavour conservation (NFC) [11]. A more general alternative is to assume the alignment in flavour space of the Yukawa matrices for each type of right-handed fermions [12]. The so-called aligned two-Higgs doublet model (A2HDM) results in a very specific structure, with all fermion-scalar interactions being proportional to the corresponding fermion masses. It also contains as particular cases the different versions of the $2 \mathrm{HDM}$ with NFC, while at the same time introduces new sources of $\mathrm{CP}$ violation beyond the Cabibbo-KobayashiMaskawa (CKM) phase $[13,14]$. These features make the A2HDM a very interesting theoretical framework, which leads to a rich and viable phenomenology, both in high-energy collider experiments [15-21], as well as in low-energy flavour physics [22-27].

In the field of rare B-meson decays, the purely leptonic processes $B_{s, d}^{0} \rightarrow \ell^{+} \ell^{-}$, with $\ell=e, \mu$ or $\tau$, play an outstanding role in testing the SM and probing physics beyond it, because they are very sensitive to the mechanism of quark-flavour mixing. Within the $\mathrm{SM}$, the FCNC transition is mediated by a one-loop amplitude, suffers from a helicitysuppression factor $m_{\ell} / m_{b}$, and is characterized by a purely leptonic final state. The first two features result in a double suppression mechanism, responsible for the extremely rare nature of these decays. The third feature implies that these processes are theoretically very clean, with the only hadronic uncertainty coming from the B-meson decay constants $f_{B_{s, d}}$. All these considerations make the rare leptonic decays $B_{s, d}^{0} \rightarrow \ell^{+} \ell^{-}$a formidable probe of physics beyond the SM, especially of models with a non-standard Higgs sector like multi-Higgs doublet models [28-36] as well as various SUSY scenarios [29, 30, 36-49].

As far as the experimental side is concerned, the decay modes with $\ell=\mu$ are especially interesting because the corresponding final state can be easily tagged. Over the last decade the upper bounds for the branching ratios of these decays have been improving continuously, thanks to the CDF and $\mathrm{D} \varnothing$ collaborations at the Tevatron and, more recently, the ATLAS, CMS and LHCb experiments at the LHC [50]. In November 2012, the LHCb experiment reported the first evidence of the decay $B_{s}^{0} \rightarrow \mu^{+} \mu^{-}$, at the $3.5 \sigma$ level [51]. The signal 
significance has been raised, respectively, to $4.0 \sigma$ and $4.3 \sigma$ by LHCb and CMS, after analyzing the currently available data set, with the averaged time-integrated branching ratio given by

$$
\overline{\mathcal{B}}\left(B_{s}^{0} \rightarrow \mu^{+} \mu^{-}\right)=\left\{\begin{array}{ll}
\left.\left.\left(2.9_{-1.0}^{+1.1} \text { (stat. }\right)_{-0.1}^{+0.3} \text { (syst. }\right)\right) \times 10^{-9} & \text { LHCb }[52] \\
\left(3.0_{-0.9}^{+1.0}\right) \times 10^{-9} & \text { CMS }[53]
\end{array},\right.
$$

where the CMS uncertainty includes both the statistical and systematic components, but is dominated by the statistical uncertainties. The two measurements lead to the weighted world average [54]

$$
\overline{\mathcal{B}}\left(B_{s}^{0} \rightarrow \mu^{+} \mu^{-}\right)_{\text {exp. }}=(2.9 \pm 0.7) \times 10^{-9} .
$$

At the same time, the branching fraction of $B_{d}^{0} \rightarrow \mu^{+} \mu^{-}$has also been determined with a signal significance of $2 \sigma$ by the two experiments:

$$
\overline{\mathcal{B}}\left(B_{d}^{0} \rightarrow \mu^{+} \mu^{-}\right)=\left\{\begin{array}{ll}
\left.\left.\left(3.7_{-2.1}^{+2.4} \text { (stat. }\right)_{-0.4}^{+0.6} \text { (syst. }\right)\right) \times 10^{-10} & \text { LHCb }[52] \\
\left(3.5_{-1.8}^{+2.1}\right) \times 10^{-10} & \text { CMS [53] }
\end{array} .\right.
$$

The corresponding combined result reads [54]

$$
\overline{\mathcal{B}}\left(B_{d}^{0} \rightarrow \mu^{+} \mu^{-}\right)_{\text {exp. }}=\left(3.6_{-1.4}^{+1.6}\right) \times 10^{-10} .
$$

These measurements are in remarkable agreement with the latest updated predictions within the SM [55]:

$$
\overline{\mathcal{B}}\left(B_{s}^{0} \rightarrow \mu^{+} \mu^{-}\right)=(3.65 \pm 0.23) \times 10^{-9}, \quad \overline{\mathcal{B}}\left(B_{d}^{0} \rightarrow \mu^{+} \mu^{-}\right)=(1.06 \pm 0.09) \times 10^{-10}
$$

where the next-to-leading order (NLO) corrections of EW origin [56], as well as the QCD corrections up to the next-to-next-to-leading order (NNLO) [57], have been taken into account. Although the experimental uncertainties are still quite large, they are expected to get significantly reduced within the next few years [58]. All these experimental and theoretical progresses will lead to new stringent constraints on physics beyond the SM.

Motivated by the above considerations, in this work we shall perform a study of the rare leptonic decays $B_{s, d}^{0} \rightarrow \ell^{+} \ell^{-}$within the A2HDM. Our paper is organized as follows. In section 2 we give a brief overview of the A2HDM Lagrangian, especially of its Yukawa and scalar sectors. In section 3 we summarize the SM results and describe the full oneloop calculation of the relevant Feynman diagrams in the A2HDM. We have performed the calculation in two different gauges, Feynman $(\xi=1)$ and unitary $(\xi=\infty)$, in order to check the gauge-independence of our results. In section 4 we discuss the impact of the model parameters on the branching ratios of these decays, taking into account the latest implications from the LHC Higgs data. Our conclusions are made in section 5. Finally, the appendix contains the explicit results for the individual Higgs-penguin diagrams. 


\section{The aligned two-Higgs doublet model}

The 2HDM extends the SM with the addition of a second scalar doublet of hypercharge $Y=\frac{1}{2}[7]$. In the so-called "Higgs basis", in which only one doublet gets a nonzero vacuum expectation value, the two doublets can be parametrized as

$$
\Phi_{1}=\left[\begin{array}{c}
G^{+} \\
\frac{1}{\sqrt{2}}\left(v+S_{1}+i G^{0}\right)
\end{array}\right], \quad \Phi_{2}=\left[\begin{array}{c}
H^{+} \\
\frac{1}{\sqrt{2}}\left(S_{2}+i S_{3}\right)
\end{array}\right],
$$

where $G^{ \pm}$and $G^{0}$ denote the Goldstone fields, and $v=\left(\sqrt{2} G_{F}\right)^{-1 / 2} \simeq 246 \mathrm{GeV}$. The five physical scalar degrees of freedom are given by the two charged fields $H^{ \pm}(x)$ and three neutral scalars $\varphi_{i}^{0}(x)=\{h(x), H(x), A(x)\}$. The latter are related with the $S_{i}$ fields through an orthogonal transformation, which is fixed by the scalar potential:

$$
\begin{aligned}
V= & \mu_{1}\left(\Phi_{1}^{\dagger} \Phi_{1}\right)+\mu_{2}\left(\Phi_{2}^{\dagger} \Phi_{2}\right)+\left[\mu_{3}\left(\Phi_{1}^{\dagger} \Phi_{2}\right)+\mu_{3}^{*}\left(\Phi_{2}^{\dagger} \Phi_{1}\right)\right] \\
& +\lambda_{1}\left(\Phi_{1}^{\dagger} \Phi_{1}\right)^{2}+\lambda_{2}\left(\Phi_{2}^{\dagger} \Phi_{2}\right)^{2}+\lambda_{3}\left(\Phi_{1}^{\dagger} \Phi_{1}\right)\left(\Phi_{2}^{\dagger} \Phi_{2}\right)+\lambda_{4}\left(\Phi_{1}^{\dagger} \Phi_{2}\right)\left(\Phi_{2}^{\dagger} \Phi_{1}\right) \\
& +\left[\left(\lambda_{5} \Phi_{1}^{\dagger} \Phi_{2}+\lambda_{6} \Phi_{1}^{\dagger} \Phi_{1}+\lambda_{7} \Phi_{2}^{\dagger} \Phi_{2}\right)\left(\Phi_{1}^{\dagger} \Phi_{2}\right)+\text { h.c. }\right] .
\end{aligned}
$$

The Hermiticity of the potential requires all parameters to be real except $\mu_{3}, \lambda_{5}, \lambda_{6}$ and $\lambda_{7}$; thus, there are 14 real parameters. The minimization conditions $\left\langle 0\left|\Phi_{1}^{T}(x)\right| 0\right\rangle=$ $(0, v / \sqrt{2})$ and $\left\langle 0\left|\Phi_{2}^{T}(x)\right| 0\right\rangle=(0,0)$ impose the relations $\mu_{1}=-\lambda_{1} v^{2}$ and $\mu_{3}=-\frac{1}{2} \lambda_{6} v^{2}$, which allow us to trade the parameters $\mu_{1}$ and $\mu_{3}$ by $v$ and $\lambda_{6}$, respectively. The freedom to rephase the field $\Phi_{2}$ implies, moreover, that only the relative phases among $\lambda_{5}, \lambda_{6}$ and $\lambda_{7}$ are physical. Therefore, we can fully characterize the potential with 11 parameters: $v$, $\mu_{2}, \lambda_{1,2,3,4},\left|\lambda_{5,6,7}\right|, \arg \left(\lambda_{5} \lambda_{6}^{*}\right)$ and $\arg \left(\lambda_{5} \lambda_{7}^{*}\right)$. Four of these parameters can be determined through the physical scalar masses.

Inserting eq. (2.1) into eq. (2.2), expanding out the resulting expression and imposing the minimization conditions, one can decompose the potential into a quadratic mass term plus cubic and quartic interactions (up to an irrelevant constant). The mass term takes the form:

$$
\begin{aligned}
V_{2} & =M_{H^{ \pm}}^{2} H^{+} H^{-}+\frac{1}{2}\left(S_{1}, S_{2}, S_{3}\right) \mathcal{M}\left(\begin{array}{c}
S_{1} \\
S_{2} \\
S_{3}
\end{array}\right) \\
& =M_{H^{ \pm}}^{2} H^{+} H^{-}+\frac{1}{2} \sum_{i=1}^{3} M_{\varphi_{i}^{0}}^{2}\left(\varphi_{i}^{0}\right)^{2}
\end{aligned}
$$

with $M_{H^{ \pm}}^{2}=\mu_{2}+\frac{1}{2} \lambda_{3} v^{2}$ and

$$
\mathcal{M}=\left(\begin{array}{ccc}
2 \lambda_{1} v^{2} & v^{2} \lambda_{6}^{\mathrm{R}} & -v^{2} \lambda_{6}^{\mathrm{I}} \\
v^{2} \lambda_{6}^{\mathrm{R}} & M_{H^{ \pm}}^{2}+v^{2}\left(\frac{\lambda_{4}}{2}+\lambda_{5}^{\mathrm{R}}\right) & -v^{2} \lambda_{5}^{\mathrm{I}} \\
-v^{2} \lambda_{6}^{\mathrm{I}} & -v^{2} \lambda_{5}^{\mathrm{I}} & M_{H^{ \pm}}^{2}+v^{2}\left(\frac{\lambda_{4}}{2}-\lambda_{5}^{\mathrm{R}}\right)
\end{array}\right),
$$

where $\lambda_{i}^{\mathrm{R}} \equiv \operatorname{Re}\left(\lambda_{i}\right)$ and $\lambda_{i}^{\mathrm{I}} \equiv \operatorname{Im}\left(\lambda_{i}\right)$. The symmetric mass matrix $\mathcal{M}$ is diagonalized by an orthogonal matrix $\mathcal{R}$, which defines the neutral mass eigenstates:

$$
\mathcal{R} \mathcal{M} \mathcal{R}^{T}=\operatorname{diag}\left(M_{h}^{2}, M_{H}^{2}, M_{A}^{2}\right), \quad \varphi_{i}^{0}=\mathcal{R}_{i j} S_{j} .
$$


In a generic case, the three mass-eigenstates $\varphi_{i}^{0}(x)$ do not have definite CP quantum numbers.

In the CP-conserving limit, $\lambda_{5}^{\mathrm{I}}=\lambda_{6}^{\mathrm{I}}=\lambda_{7}^{\mathrm{I}}=0$ and $S_{3}$ does not mix with the other two neutral fields. The scalar spectrum contains then a CP-odd field $A=S_{3}$ and two CP-even scalars $h$ and $H$ which mix through the two-dimensional rotation matrix: ${ }^{1}$

$$
\left(\begin{array}{c}
h \\
H
\end{array}\right)=\left[\begin{array}{cc}
\cos \tilde{\alpha} & \sin \tilde{\alpha} \\
-\sin \tilde{\alpha} & \cos \tilde{\alpha}
\end{array}\right]\left(\begin{array}{l}
S_{1} \\
S_{2}
\end{array}\right) .
$$

We shall adopt the conventions $M_{h} \leq M_{H}$ and $0 \leq \tilde{\alpha} \leq \pi$, so that $\sin \tilde{\alpha}$ is always positive. The masses of the three physical neutral scalars are given in this case by

$$
M_{h}^{2}=\frac{1}{2}(\Sigma-\Delta), \quad M_{H}^{2}=\frac{1}{2}(\Sigma+\Delta), \quad M_{A}^{2}=M_{H^{ \pm}}^{2}+v^{2}\left(\frac{\lambda_{4}}{2}-\lambda_{5}^{\mathrm{R}}\right),
$$

where

$$
\begin{aligned}
& \Sigma=M_{H^{ \pm}}^{2}+v^{2}\left(2 \lambda_{1}+\frac{\lambda_{4}}{2}+\lambda_{5}^{\mathrm{R}}\right), \\
& \Delta=\sqrt{\left[M_{H^{ \pm}}^{2}+v^{2}\left(-2 \lambda_{1}+\frac{\lambda_{4}}{2}+\lambda_{5}^{\mathrm{R}}\right)\right]^{2}+4 v^{4}\left(\lambda_{6}^{\mathrm{R}}\right)^{2}}=-\frac{2 v^{2} \lambda_{6}^{\mathrm{R}}}{\sin (2 \tilde{\alpha})},
\end{aligned}
$$

and the mixing angle is determined through

$$
\tan \tilde{\alpha}=\frac{M_{h}^{2}-2 \lambda_{1} v^{2}}{v^{2} \lambda_{6}^{\mathrm{R}}}=\frac{v^{2} \lambda_{6}^{\mathrm{R}}}{2 \lambda_{1} v^{2}-M_{H}^{2}} .
$$

The cubic and quartic self-couplings among the physical scalars and their interactions with the gauge bosons can be derived straightforwardly. Their explicit form could be found, for example, in refs. [8, 9, 20, 21, 59-62].

\subsection{Yukawa sector}

In the Higgs basis, the most generic Yukawa Lagrangian of the 2HDM is given by

$$
\mathcal{L}_{Y}=-\frac{\sqrt{2}}{v}\left[\bar{Q}_{L}^{\prime}\left(M_{d}^{\prime} \Phi_{1}+Y_{d}^{\prime} \Phi_{2}\right) d_{R}^{\prime}+\bar{Q}_{L}^{\prime}\left(M_{u}^{\prime} \tilde{\Phi}_{1}+Y_{u}^{\prime} \tilde{\Phi}_{2}\right) u_{R}^{\prime}+\bar{L}_{L}^{\prime}\left(M_{\ell}^{\prime} \Phi_{1}+Y_{\ell}^{\prime} \Phi_{2}\right) \ell_{R}^{\prime}\right]+\text { h.c. },
$$

where $\tilde{\Phi}_{i}(x)=i \tau_{2} \Phi_{i}^{*}(x)$ are the charge-conjugated scalar doublets with hypercharge $Y=$ $-\frac{1}{2}, Q_{L}^{\prime}$ and $L_{L}^{\prime}$ denote the SM left-handed quark and lepton doublets, respectively, and $u_{R}^{\prime}, d_{R}^{\prime}$ and $\ell_{R}^{\prime}$ are the corresponding right-handed singlets, in the weak interaction basis. All fermionic fields are written as 3 -vectors in flavour space and, accordingly, the couplings $M_{f}^{\prime}$ and $Y_{f}^{\prime}(f=u, d, \ell)$ are $3 \times 3$ complex matrices.

In general, the Yukawa matrices $M_{f}^{\prime}$ and $Y_{f}^{\prime}$ cannot be simultaneously diagonalized in flavour space. Thus, in the fermion mass-eigenstate basis with diagonal mass matrices $M_{f}$, the corresponding Yukawa matrices $Y_{f}$ remain non-diagonal, giving rise to tree-level

\footnotetext{
${ }^{1}$ The scalar mixing is often parametrized in terms of $\alpha^{\prime}=\tilde{\alpha}+\pi / 2$, so that the SM limit corresponds to $\alpha^{\prime}=\pi / 2[8,9]$. We prefer to describe small deviations from the SM limit with $\tilde{\alpha} \simeq 0$.
} 


\begin{tabular}{|c|c|c|c|}
\hline Model & $\varsigma_{d}$ & $\varsigma_{u}$ & $\varsigma_{l}$ \\
\hline Type I & $\cot \beta$ & $\cot \beta$ & $\cot \beta$ \\
Type II & $-\tan \beta$ & $\cot \beta$ & $-\tan \beta$ \\
Type X (lepton-specific) & $\cot \beta$ & $\cot \beta$ & $-\tan \beta$ \\
Type Y (flipped) & $-\tan \beta$ & $\cot \beta$ & $\cot \beta$ \\
Inert & 0 & 0 & 0 \\
\hline
\end{tabular}

Table 1. The one-to-one correspondence between different specific choices of the couplings $\varsigma_{f}$ and the $2 \mathrm{HDMs}$ based on discrete $\mathcal{Z}_{2}$ symmetries.

FCNC interactions. In the A2HDM, the tree-level FCNCs are eliminated by requiring the alignment in flavour space of the two Yukawa matrices coupling to a given type of right-handed fermions [12]

$$
Y_{d, \ell}=\varsigma_{d, \ell} M_{d, \ell}, \quad Y_{u}=\varsigma_{u}^{*} M_{u},
$$

where the three proportionality parameters $\varsigma_{f}(f=d, u, \ell)$ are arbitrary complex numbers and introduce new sources of $\mathrm{CP}$ violation. The Yukawa interactions of the physical scalars with the fermion mass-eigenstate fields then read [12]

$$
\begin{aligned}
\mathcal{L}_{Y}= & -\frac{\sqrt{2}}{v} H^{+}\left\{\bar{u}\left[\varsigma_{d} V M_{d} P_{R}-\varsigma_{u} M_{u}^{\dagger} V P_{L}\right] d+\varsigma_{\ell} \bar{\nu} M_{\ell} P_{R} \ell\right\} \\
& -\frac{1}{v} \sum_{\varphi_{i}^{0}, f} y_{f}^{\varphi_{i}^{0}} \varphi_{i}^{0}\left[\bar{f} M_{f} P_{R} f\right]+\text { h.c. },
\end{aligned}
$$

where $P_{R, L} \equiv \frac{1 \pm \gamma_{5}}{2}$ are the right-handed and left-handed chirality projectors, $M_{f}$ the diagonal fermion mass matrices, and $V$ the CKM quark-mixing matrix [13, 14]. The couplings of the neutral scalar fields to fermion pairs are given by

$$
y_{d, \ell}^{\varphi_{i}^{0}}=\mathcal{R}_{i 1}+\left(\mathcal{R}_{i 2}+i \mathcal{R}_{i 3}\right) \varsigma_{d, \ell}, \quad y_{u}^{\varphi_{i}^{0}}=\mathcal{R}_{i 1}+\left(\mathcal{R}_{i 2}-i \mathcal{R}_{i 3}\right) \varsigma_{u}^{*} .
$$

In the A2HDM, all fermionic couplings to scalars are proportional to the corresponding fermion masses, and the only source of flavour-changing interactions is the CKM quarkmixing matrix $V$, while all leptonic couplings and the quark neutral-current interactions are diagonal in flavour. All possible freedom allowed by the alignment conditions is encoded by the three family-universal complex parameters $\varsigma_{f}$, which provide new sources of CP violation without tree-level FCNCs [12]. The usual models with NFC, based on discrete $\mathcal{Z}_{2}$ symmetries, are recovered for particular values of the couplings $\varsigma_{f}$, as indicated in table 1. Explicit examples of symmetry-protected underlying theories leading to a lowenergy A2HDM structure have been discussed in ref. [63-65].

The alignment conditions in eq. (2.12) presumably hold at some high-energy scale $\Lambda_{A}$ and are spoiled by radiative corrections. These higher-order contributions induce a misalignment of the Yukawa matrices, generating small FCNC effects suppressed by the corresponding loop factors [12, 22, 66-70]. However, the flavour symmetries of the A2HDM tightly constrain the possible FCNC structures, keeping their effects well below the present 
experimental bounds [22-27]. Using the renormalization-group equations (RGEs) [67-70], one can check that the only FCNC local structures induced at one loop take the form $[22,66]$

$$
\begin{aligned}
\mathcal{L}_{\mathrm{FCNC}}= & \frac{\mathcal{C}}{4 \pi^{2} v^{3}}\left(1+\varsigma_{u}^{*} \varsigma_{d}\right) \sum_{i} \varphi_{i}^{0}\left\{\left(\mathcal{R}_{i 2}+i \mathcal{R}_{i 3}\right)\left(\varsigma_{d}-\varsigma_{u}\right)\left[\bar{d}_{L} V^{\dagger} M_{u} M_{u}^{\dagger} V M_{d} d_{R}\right]\right. \\
& \left.-\left(\mathcal{R}_{i 2}-i \mathcal{R}_{i 3}\right)\left(\varsigma_{d}^{*}-\varsigma_{u}^{*}\right)\left[\bar{u}_{L} V M_{d} M_{d}^{\dagger} V^{\dagger} M_{u} u_{R}\right]\right\}+ \text { h.c. },
\end{aligned}
$$

which vanishes identically when $\varsigma_{d}=\varsigma_{u}\left(\mathcal{Z}_{2}\right.$ models of types I, X and inert) or $\varsigma_{d}=$ $-1 / \varsigma_{u}^{*}$ (types II and $\mathrm{Y}$ ), as it should be.

Although the numerical effect of the local term in eq. (2.15) is suppressed by $m_{q} m_{q^{\prime}}^{2} / v^{3}$ and quark-mixing factors, its tree-level contribution is needed to render finite the contribution from one-loop Higgs-penguin diagrams to $B_{s, d}^{0} \rightarrow \ell^{+} \ell^{-}$, as will be detailed later. The renormalization of the coupling constant $\mathcal{C}$ is determined to be

$$
\mathcal{C}=\mathcal{C}_{R}(\mu)+\frac{1}{2}\left\{\frac{2 \mu^{D-4}}{D-4}+\gamma_{E}-\ln (4 \pi)\right\}
$$

where $D$ is the space-time dimension. Thus, the renormalized coupling satisfies

$$
\mathcal{C}_{R}(\mu)=\mathcal{C}_{R}\left(\mu_{0}\right)-\ln \left(\mu / \mu_{0}\right)
$$

Assuming the alignment to be exact at the scale $\Lambda_{A}$, i.e., $\mathcal{C}_{R}\left(\Lambda_{A}\right)=0$, this implies $\mathcal{C}_{R}(\mu)=$ $\ln \left(\Lambda_{A} / \mu\right)$.

\section{Calculation of $\overline{\mathcal{B}}\left(B_{s, d}^{0} \rightarrow \ell^{+} \ell^{-}\right)$}

\subsection{Effective Hamiltonian}

The rare leptonic $B_{s, d}^{0} \rightarrow \ell^{+} \ell^{-}$decays proceed through loop diagrams in both the SM and the A2HDM. After decoupling the heavy degrees of freedom, including the top quark, the weak gauge bosons, as well as the charged and neutral Higgs bosons, these decays are described by a low-energy effective Hamiltonian [71-74]

$$
\mathcal{H}_{\mathrm{eff}}=-\frac{G_{F} \alpha}{\sqrt{2} \pi s_{W}^{2}}\left[V_{t b} V_{t q}^{*} \sum_{i}^{10, S, P}\left(C_{i} \mathcal{O}_{i}+C_{i}^{\prime} \mathcal{O}_{i}^{\prime}\right)+\text { h.c. }\right],
$$

where $G_{F}$ is the Fermi coupling constant, $\alpha=e^{2} / 4 \pi$ the QED fine-structure constant, and $s_{W}=\sin \theta_{W}$ the sine of the weak angle. The effective four-fermion operators are given, respectively, as

$$
\begin{aligned}
\mathcal{O}_{10} & =\left(\bar{q} \gamma_{\mu} P_{L} b\right)\left(\bar{\ell} \gamma^{\mu} \gamma_{5} \ell\right), & \mathcal{O}_{10}^{\prime} & =\left(\bar{q} \gamma_{\mu} P_{R} b\right)\left(\bar{\ell} \gamma^{\mu} \gamma_{5} \ell\right), \\
\mathcal{O}_{S} & =\frac{m_{\ell} m_{b}}{M_{W}^{2}}\left(\bar{q} P_{R} b\right)(\bar{\ell} \ell), & \mathcal{O}_{S}^{\prime} & =\frac{m_{\ell} m_{b}}{M_{W}^{2}}\left(\bar{q} P_{L} b\right)(\bar{\ell} \ell), \\
\mathcal{O}_{P} & =\frac{m_{\ell} m_{b}}{M_{W}^{2}}\left(\bar{q} P_{R} b\right)\left(\bar{\ell} \gamma_{5} \ell\right), & \mathcal{O}_{P}^{\prime} & =\frac{m_{\ell} m_{b}}{M_{W}^{2}}\left(\bar{q} P_{L} b\right)\left(\bar{\ell} \gamma_{5} \ell\right),
\end{aligned}
$$

where $\ell=e, \mu, \tau ; q=d, s$, and $m_{b}=m_{b}(\mu)$ denotes the $b$-quark running mass in the modified minimal subtraction $(\overline{\mathrm{MS}})$ scheme. In this paper, we shall neglect the operators $\mathcal{O}_{i}^{\prime}$, 
because they only give contributions proportional to the light-quark mass $m_{q}$. Operators involving the vector current $\bar{\ell} \gamma^{\mu} \ell$ do not contribute to $B_{s, d}^{0} \rightarrow \ell^{+} \ell^{-}$because the conserved vector current vanishes when contracted with the $B_{q}^{0}$ momentum. Since the matrix element $\left\langle 0\left|\bar{q} \sigma_{\mu \nu} b\right| \bar{B}_{q}^{0}(p)\right\rangle=0$, there is also no contribution from the tensor operators. Thus, only the operators $\mathcal{O}_{10}, \mathcal{O}_{S}$ and $\mathcal{O}_{P}$ survive in our approximation.

As there are highly separated mass scales in the decays $B_{s, d}^{0} \rightarrow \ell^{+} \ell^{-}$, short-distance QCD corrections can contain large logarithms like $\ln \left(\mu_{b} / M_{W}\right)$ with $\mu_{b} \sim \mathcal{O}\left(m_{b}\right)$, which must be summed up to all orders in perturbation theory with the help of renormalizationgroup techniques. The evolution of the Wilson coefficients from the scale $\mathcal{O}\left(M_{W}\right)$ down to $\mathcal{O}\left(\mu_{b}\right)$ requires the solution of the RGEs of the corresponding operators $\mathcal{O}_{10}, \mathcal{O}_{S}$ and $\mathcal{O}_{P}$. However, the operator $\mathcal{O}_{10}$ has zero anomalous dimension due to the conservation of the $(V-A)$ quark current in the limit of vanishing quark masses. The operators $\mathcal{O}_{S}$ and $\mathcal{O}_{P}$ have also zero anomalous dimension, because the anomalous dimensions of the $b$-quark mass $m_{b}(\mu)$ and the scalar current $\left(\bar{q} P_{R} b\right)(\mu)$ cancel each other. Thus, with the operators defined by eq. (3.2), the corresponding Wilson coefficients do not receive additional renormalization due to QCD corrections.

In the SM, the contributions from the scalar and pseudoscalar operators are quite suppressed and, therefore, are usually neglected in phenomenological analyses. However, they can be much more sizeable in models with enlarged Higgs sectors, such as the A2HDM, especially when the Yukawa and/or scalar-potential couplings are large. Therefore, the $B_{s, d}^{0} \rightarrow \ell^{+} \ell^{-}$data provide useful constraints on the model parameters. To get the theoretical predictions for $\overline{\mathcal{B}}\left(B_{s, d}^{0} \rightarrow \ell^{+} \ell^{-}\right)$, the main task is then to calculate the three Wilson coefficients $C_{10, S, P}$ in both the SM and the A2HDM, details of which will be presented in the next few subsections.

\subsection{Computational method}

The standard way to find the Wilson coefficients is to require equality of one-particle irreducible amputated Green functions calculated in the full and in the effective theory $[75,76]$. The former requires the calculation of various box, penguin and self-energy diagrams. We firstly use the program FeynArts [77], with the model files provided by the package FeynRules [78-80], to generate all the Feynman diagrams contributing to the decays $B_{s, d}^{0} \rightarrow \ell^{+} \ell^{-}$, as well as the corresponding amplitudes, which can then be evaluated straightforwardly.

Throughout the whole calculation, we set the light-quark masses $m_{d, s}$ to zero; while for $m_{b}$, we keep it up to linear order. As the external momenta are much smaller than the masses of internal top-quark, gauge bosons, as well as charged and neutral scalars, the Feynman integrands are expanded in external momenta before performing the loop integration $[81,82]$

$$
\frac{1}{(k+l)^{2}-M^{2}}=\frac{1}{k^{2}-M^{2}}\left[1-\frac{l^{2}+2(k \cdot l)}{k^{2}-M^{2}}+\frac{4(k \cdot l)^{2}}{\left(k^{2}-M^{2}\right)^{2}}\right]+\mathcal{O}\left(l^{4} / M^{4}\right),
$$

where $k$ denotes the loop momentum, $M$ a heavy mass and $l$ an arbitrary external momentum. In addition, we employ the naive dimensional regularization scheme with an 
anti-commuting $\gamma_{5}$ to regularize the divergences appearing in Feynman integrals. After the Taylor expansion and factorizing out the external momenta, the integrals remain dependent only on the loop momentum and the heavy masses $M$. Subsequently, we apply the partial fraction decomposition [83]

$$
\frac{1}{\left(q^{2}-m_{1}^{2}\right)\left(q^{2}-m_{2}^{2}\right)}=\frac{1}{m_{1}^{2}-m_{2}^{2}}\left[\frac{1}{q^{2}-m_{1}^{2}}-\frac{1}{q^{2}-m_{2}^{2}}\right]
$$

which allows a reduction of all the Feynman integrals to those in which only a single mass parameter occurs in the propagator denominators. Finally, after reduction of tensor integrals to scalar ones, the only non-vanishing one-loop integrals take the form [84]

$$
\int \frac{d^{D} k}{(2 \pi)^{D}} \frac{1}{\left(k^{2}-m^{2}\right)^{n}}=\frac{(-1)^{n} i}{(4 \pi)^{D / 2}} \frac{\Gamma(n-D / 2)}{\Gamma(n)}\left(\frac{1}{m^{2}}\right)^{n-D / 2},
$$

with an arbitrary integer power $n$ and with $m \neq 0$.

The computational procedure has also been checked through an independent analytic calculation of the Feynman diagrams, using more standard techniques such as the Feynman parametrization to combine propagators. We found full agreement between the results obtained with these two methods.

It should be noted that, in deriving the effective Hamiltonian in eq. (3.1), the limit $m_{u, c} \rightarrow 0$ and the unitarity of the CKM matrix,

$$
V_{u q}^{*} V_{u b}+V_{c q}^{*} V_{c b}+V_{t q}^{*} V_{t b}=0,
$$

have been implicitly exploited. In general, the Wilson coefficients $C_{i}$ are functions of the internal up-type quark masses, together with the corresponding CKM factors [75, 76]:

$$
C_{i}=\sum_{j=u, c, t} V_{j q}^{*} V_{j b} F_{i}\left(x_{j}\right),
$$

where $x_{j}=m_{j}^{2} / M_{W}^{2}$, and $F_{i}\left(x_{j}\right)$ denote the loop functions. The unitarity relation in eq. (3.6) implies vanishing coefficients $C_{i}$ if the internal quark masses are set to be equal, i.e., $x_{u}=x_{c}=x_{t}$. For this reason, we need only to calculate explicitly the contributions from internal top quarks, while those from up and charm quarks are taken into account by means of simply omitting the mass-independent terms in the basic functions $F_{i}\left(x_{t}\right)$. For simplicity, we also introduce the following mass ratios:

$$
x_{t}=\frac{m_{t}^{2}}{M_{W}^{2}}, \quad x_{H^{+}}=\frac{M_{H^{ \pm}}^{2}}{M_{W}^{2}}, \quad x_{\varphi_{i}^{0}}=\frac{M_{\varphi_{i}^{0}}^{2}}{M_{W}^{2}}, \quad x_{h_{\mathrm{SM}}}=\frac{M_{h_{\mathrm{SM}}}^{2}}{M_{W}^{2}},
$$

where $m_{t}=m_{t}(\mu)$ is the top-quark running mass in the $\overline{\mathrm{MS}}$ scheme, and $h_{\mathrm{SM}}$ the SM Higgs boson.

In order to make a detailed presentation of our results, we shall split the different contributions to the Wilson coefficients into the form:

$$
\begin{aligned}
C_{10}= & C_{10}^{\mathrm{SM}}+C_{10}^{\mathrm{Z} \text { penguin, } \mathrm{A} 2 \mathrm{HDM}}, \\
C_{S}= & C_{S}^{\mathrm{box}, \mathrm{SM}}+C_{S}^{\mathrm{box}, \mathrm{A} 2 \mathrm{HDM}}+C_{S}^{\varphi_{i}^{0}, \mathrm{~A} 2 \mathrm{HDM}}, \\
C_{P}= & C_{P}^{\mathrm{box}, \mathrm{SM}}+C_{P}^{\mathrm{Z} \text { penguin, } \mathrm{SM}}+C_{P}^{\mathrm{GB} \text { penguin, } \mathrm{SM}}+C_{P}^{\mathrm{box}, \mathrm{A} 2 \mathrm{HDM}} \\
& +C_{P}^{\mathrm{Z} \text { penguin, } \mathrm{A} 2 \mathrm{HDM}}+C_{P}^{\mathrm{GB} \text { penguin, } \mathrm{A} 2 \mathrm{HDM}}+C_{P}^{\varphi_{i}^{0}, \mathrm{~A} 2 \mathrm{HDM}} .
\end{aligned}
$$



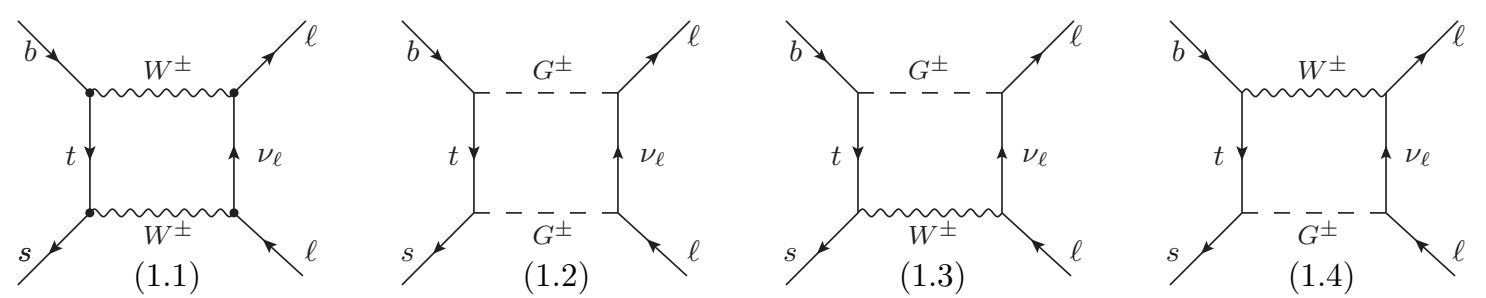

Figure 1. SM $W$-box diagrams contributing to $\bar{B}_{s}^{0} \rightarrow \ell^{+} \ell^{-}$. Diagrams involving Goldstone bosons $G^{ \pm}$are absent in the unitary gauge.

The pieces labeled with "SM" only involve SM fields (without the Higgs), while those denoted by "A2HDM" contain the scalar contributions. We have calculated all the individual diagrams in both the Feynman $(\xi=1)$ and the unitary $(\xi=\infty)$ gauges. Goldstone boson (GB) contributions are of course absent in the unitary gauge. While the contributions of the box and penguin diagrams to the Wilson coefficients are separately gauge dependent, their sum is indeed independent of the EW gauge fixing $[85,86]$. Note that photonic penguin diagrams, in both the SM and the A2HDM, do not contribute to the decays $B_{s, d}^{0} \rightarrow \ell^{+} \ell^{-}$because of the pure vector nature of the electromagnetic leptonic coupling.

In $B_{s, d}^{0} \rightarrow \ell^{+} \ell^{-}$the external momenta are small compared to the EW scale $M_{W}$. One can then set all external momenta to zero when evaluating $C_{10}$. However, the external momenta must be taken into account to evaluate the scalar Wilson coefficients $C_{S}$ and $C_{P}$, otherwise some contributions would be missed.

\subsection{Wilson coefficients in the SM}

In the SM, the dominant contributions to the decays $B_{s, d}^{0} \rightarrow \ell^{+} \ell^{-}$come from the $W$-box and $Z$-penguin diagrams shown in figures 1 and 2, respectively, which generate the Wilson coefficient:

$$
C_{10}^{\mathrm{SM}}=-\eta_{Y}^{\mathrm{EW}} \eta_{Y}^{\mathrm{QCD}} Y_{0}\left(x_{t}\right)
$$

where

$$
Y_{0}\left(x_{t}\right)=\frac{x_{t}}{8}\left[\frac{x_{t}-4}{x_{t}-1}+\frac{3 x_{t}}{\left(x_{t}-1\right)^{2}} \ln x_{t}\right]
$$

is the one-loop function that was calculated for the first time in ref. [87]. The factor $\eta_{Y}^{\mathrm{EW}}$ accounts for both the NLO EW matching corrections [56], as well as the logarithmically enhanced QED corrections that originate from the renormalization group evolution [55, 57], while the coefficient $\eta_{Y}^{\mathrm{QCD}}$ stands for the NLO [88-91] and NNLO [57] QCD corrections.

When the small external momenta are taken into account, the SM $W$-box and $Z$ penguin diagrams also generate contributions to the Wilson coefficients $C_{S}$ and $C_{P}$. The contribution from diagram 1.2 can be neglected, because it contains two leptonic Goldstone couplings which generate a suppression factor $m_{\ell}^{2} / M_{W}^{2}$. The scalar contribution from the 

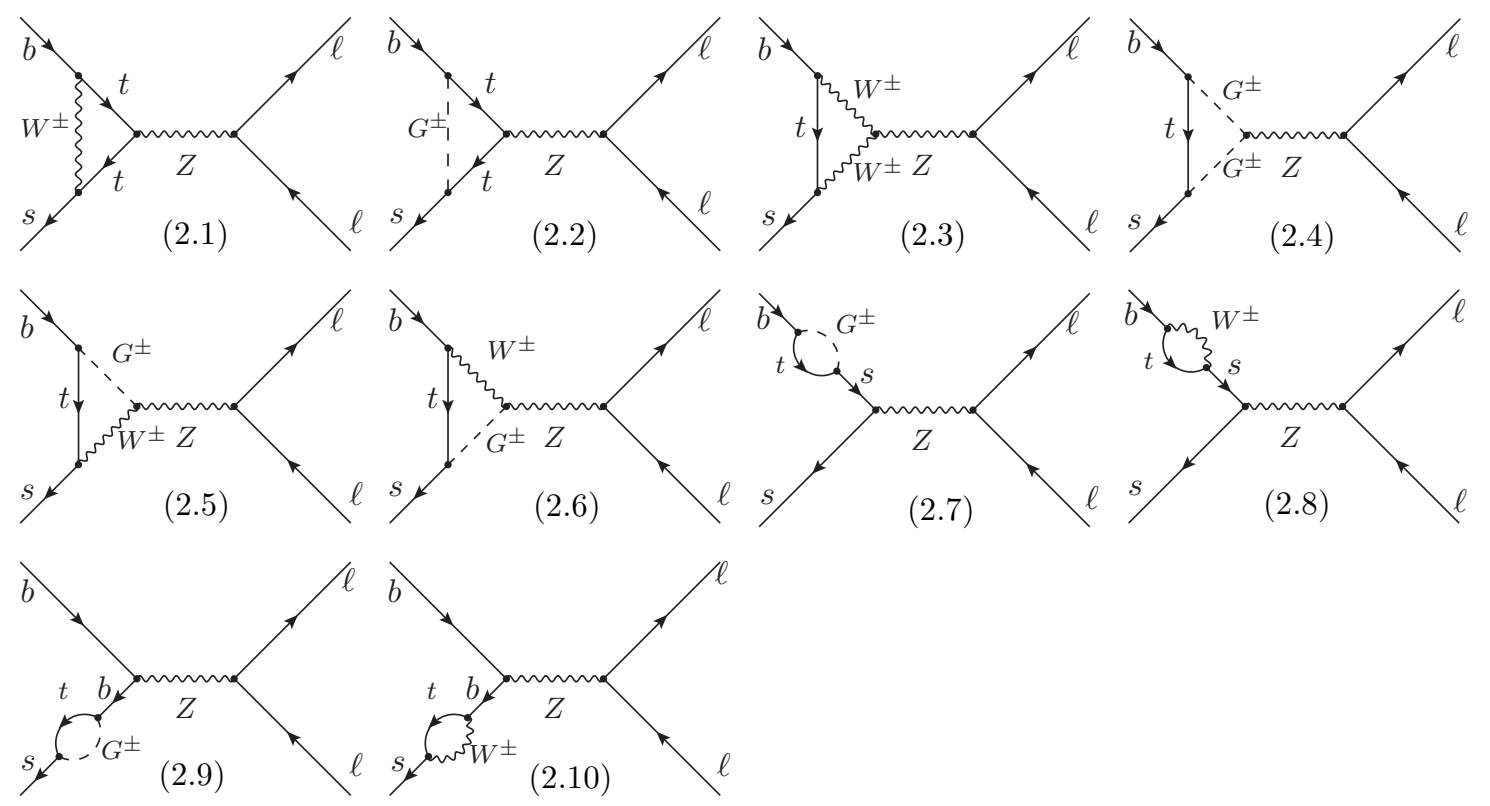

$(2.7)$

$(2.8)$

Figure 2. SM $Z$-penguin diagrams contributing to $\bar{B}_{s}^{0} \rightarrow \ell^{+} \ell^{-}$. Diagrams involving Goldstone bosons $G^{ \pm}$are absent in the unitary gauge.

remaining box diagrams is given by:

$$
\begin{aligned}
C_{S, \text { Feynman }}^{\text {box } \mathrm{SM}} & =-\frac{x_{t}\left(x_{t}-2\right)}{12\left(x_{t}-1\right)^{2}}+\frac{\left(x_{t}-2\right)\left(3 x_{t}-1\right)}{24\left(x_{t}-1\right)^{3}} \ln x_{t}, \\
C_{S, \text { Unitary }}^{\text {box SM }} & =-\frac{x_{t}\left(x_{t}+1\right)}{48\left(x_{t}-1\right)^{2}}-\frac{\left(x_{t}-2\right)\left(3 x_{t}^{2}-3 x_{t}+1\right)}{24\left(x_{t}-1\right)^{3}} \ln x_{t},
\end{aligned}
$$

where the two different expressions correspond to the results obtained in the Feynman and unitary gauges, respectively.

In the SM there is an additional contribution to the scalar Wilson coefficient $C_{S}$ from the Higgs-penguin diagrams shown in figure 3 , which is by itself gauge dependent [86, 92, 93] and should cancel the gauge dependence of the $W$-box contribution. We find the result:

$$
\begin{aligned}
C_{S, \text { Feynman }}^{\mathrm{h} \text { penguin, SM }} & =-\frac{x_{t}}{8}\left[\frac{3}{x_{h_{\mathrm{SM}}}}-\frac{x_{t}-3}{2\left(x_{t}-1\right)^{2}}+\frac{x_{t}\left(x_{t}-2\right)}{\left(x_{t}-1\right)^{3}} \ln x_{t}\right], \\
C_{S, \text { Unitary }}^{\mathrm{h} \text { penguin, SM }} & =-\frac{3 x_{t}}{8 x_{h_{\mathrm{SM}}}} .
\end{aligned}
$$

The sum of the two contributions to $C_{S}$ is indeed gauge independent:

$$
\begin{aligned}
C_{S}^{\mathrm{SM}} & =C_{S, \text { Feynman }}^{\text {box }, \mathrm{SM}}+C_{S, \text { Feynman }}^{\mathrm{h} \text { penguin, } \mathrm{SM}}=C_{S, \text { Unitary }}^{\mathrm{box}, \mathrm{SM}}+C_{S, \text { Unitary }}^{\mathrm{h} \text { penguin } \mathrm{SM}} \\
& =-\frac{3 x_{t}}{8 x_{h_{\mathrm{SM}}}}-\frac{x_{t}\left(x_{t}+1\right)}{48\left(x_{t}-1\right)^{2}}-\frac{\left(x_{t}-2\right)\left(3 x_{t}^{2}-3 x_{t}+1\right)}{24\left(x_{t}-1\right)^{3}} \ln x_{t} .
\end{aligned}
$$



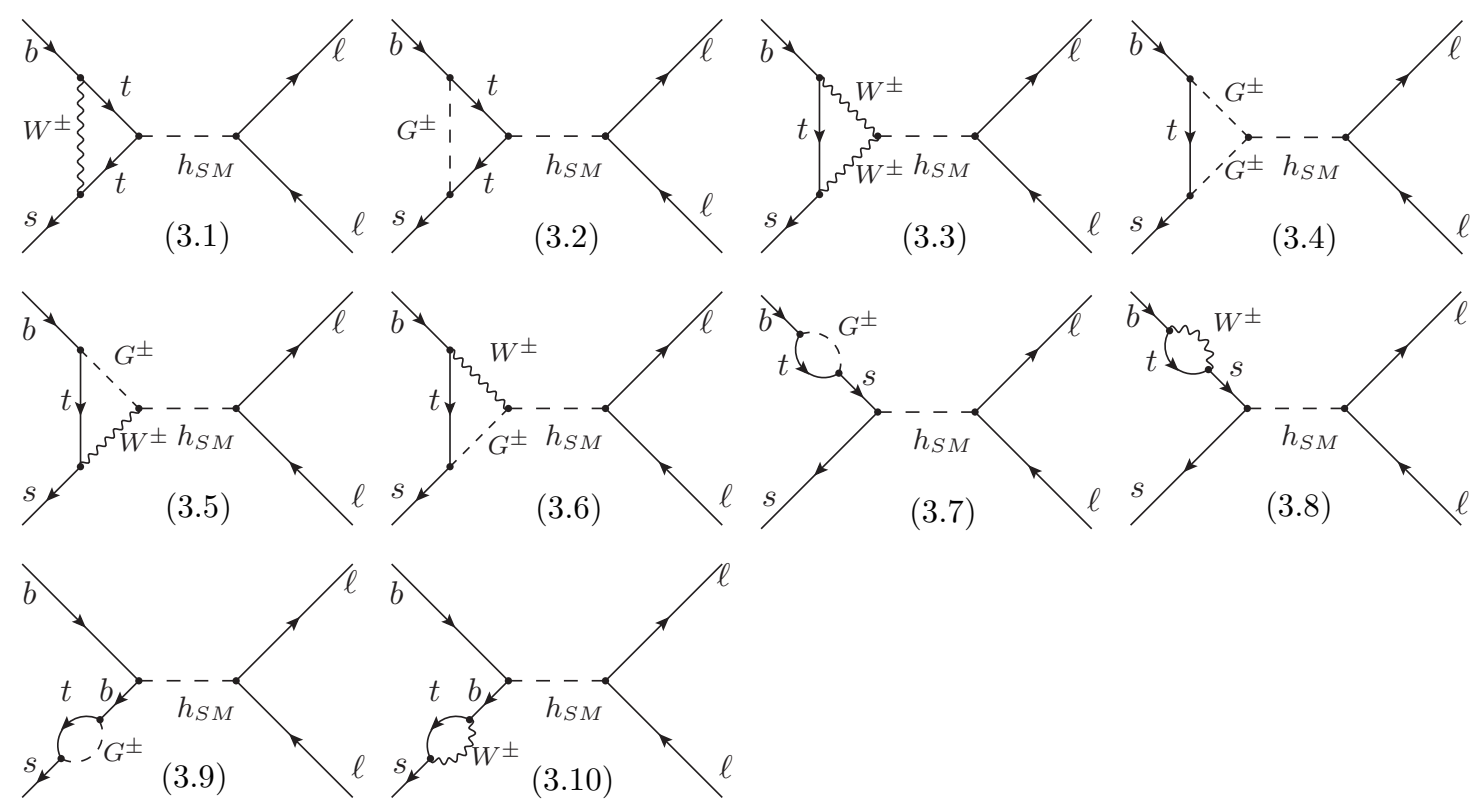

Figure 3. SM Higgs-penguin diagrams contributing to $\bar{B}_{s}^{0} \rightarrow \ell^{+} \ell^{-}$. Contributions with Goldstone bosons $G^{ \pm}$are absent in the unitary gauge.
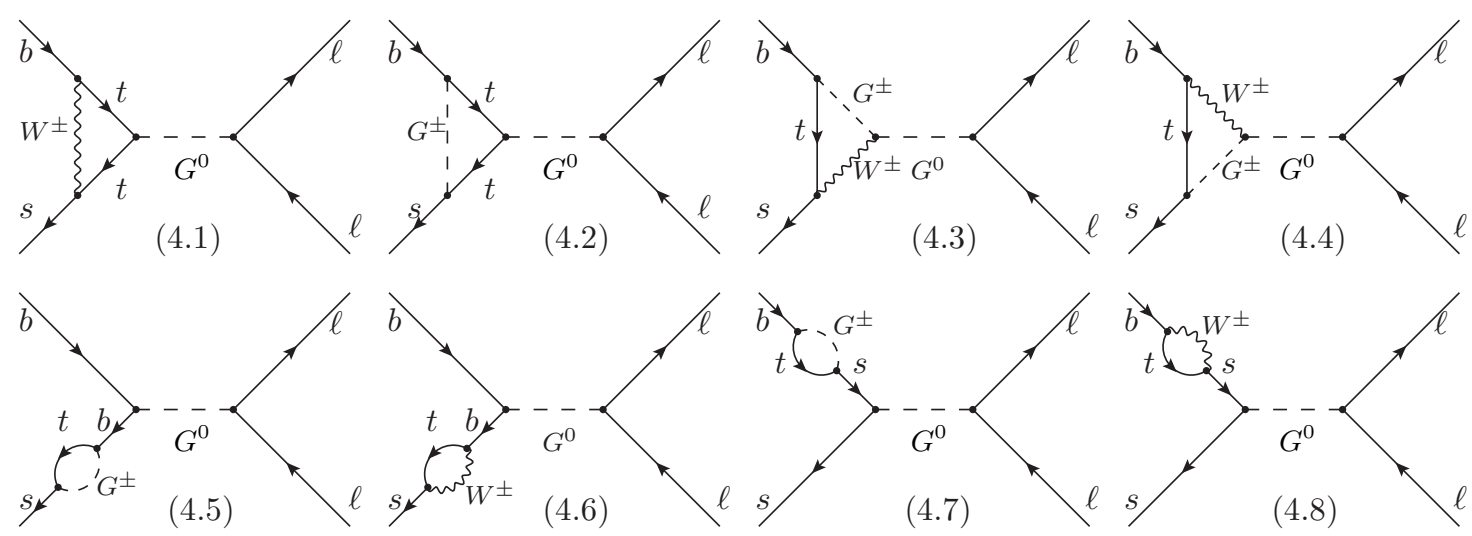

Figure 4. SM Goldstone-penguin diagrams contributing to $\bar{B}_{s}^{0} \rightarrow \ell^{+} \ell^{-}$. These contributions are absent in the unitary gauge.

The contribution from the SM $W$-box diagrams (figure 1) to the pseudoscalar Wilson coefficient $C_{P}$ is given by:

$$
\begin{aligned}
C_{P, \text { Feynman }}^{\text {box SM }} & =\frac{x_{t}\left(35 x_{t}^{2}-82 x_{t}-1\right)}{72\left(x_{t}-1\right)^{3}}-\frac{9 x_{t}^{3}-28 x_{t}^{2}+x_{t}+2}{24\left(x_{t}-1\right)^{4}} \ln x_{t}, \\
C_{P, \text { Unitary }}^{\text {box, SM }} & =\frac{x_{t}\left(71 x_{t}^{2}-172 x_{t}-19\right)}{144\left(x_{t}-1\right)^{3}}+\frac{x_{t}^{4}-12 x_{t}^{3}+34 x_{t}^{2}-x_{t}-2}{24\left(x_{t}-1\right)^{4}} \ln x_{t} .
\end{aligned}
$$

Additional contributions to $C_{P}$ are generated by the $Z$ - and Goldstone-penguin diagrams shown in figures 2 and 4, respectively. The contributions from diagrams 4.6, 4.7 
and 4.8 are proportional to the light-quark mass and can be therefore neglected. We find:

$$
\begin{aligned}
C_{P, \text { Feynman }}^{\mathrm{Z} \text { penguin, } \mathrm{SM}}= & \frac{x_{t}\left(5 x_{t}^{2}+16 x_{t}+3\right)}{48\left(x_{t}-1\right)^{3}}-\frac{x_{t}^{4}+x_{t}^{3}+18 x_{t}^{2}-12 x_{t}+4}{24\left(x_{t}-1\right)^{4}} \ln x_{t} \\
& -s_{W}^{2}\left[\frac{x_{t}\left(5 x_{t}^{2}+40 x_{t}-21\right)}{72\left(x_{t}-1\right)^{3}}-\frac{3 x_{t}^{4}-3 x_{t}^{3}+36 x_{t}^{2}-32 x_{t}+8}{36\left(x_{t}-1\right)^{4}} \ln x_{t}\right], \\
C_{P, \text { Feynman }}^{\text {GB penguin } \mathrm{SM}}= & \left(1-s_{W}^{2}\right) \frac{x_{t}}{4}\left[\frac{x_{t}-6}{x_{t}-1}+\frac{3 x_{t}+2}{\left(x_{t}-1\right)^{2}} \ln x_{t}\right]
\end{aligned}
$$

and

$$
\begin{array}{r}
C_{P, \text { Unitary }}^{Z \text { penguin, }}=\frac{1}{12}\left[\frac{x_{t}\left(18 x_{t}^{3}-137 x_{t}^{2}+262 x_{t}-95\right)}{6\left(x_{t}-1\right)^{3}}+\frac{8 x_{t}^{4}-11 x_{t}^{3}-15 x_{t}^{2}+12 x_{t}-2}{\left(x_{t}-1\right)^{4}} \ln x_{t}\right] \\
-\frac{s_{W}^{2}}{36}\left[\frac{x_{t}\left(18 x_{t}^{3}-139 x_{t}^{2}+274 x_{t}-129\right)}{2\left(x_{t}-1\right)^{3}}+\frac{24 x_{t}^{4}-33 x_{t}^{3}-45 x_{t}^{2}+50 x_{t}-8}{\left(x_{t}-1\right)^{4}} \ln x_{t}\right] .
\end{array}
$$

Using the above results, one can easily check that the SM contribution to $C_{P}$ is also gauge independent:

$$
\begin{aligned}
C_{P}^{\mathrm{SM}}= & C_{P, \text { Feynman }}^{\text {box, SM }}+C_{P, \text { Feynman }}^{\text {Z penguin, SM }}+C_{P, \text { Feynman }}^{\mathrm{GB} \text { penguin } \mathrm{SM}}=C_{P, \text { Unitary }}^{\text {box, SM }}+C_{P, \text { Unitary }}^{\text {Z penguin, SM }} \\
= & \frac{1}{24}\left[\frac{x_{t}\left(36 x_{t}^{3}-203 x_{t}^{2}+352 x_{t}-209\right)}{6\left(x_{t}-1\right)^{3}}+\frac{17 x_{t}^{4}-34 x_{t}^{3}+4 x_{t}^{2}+23 x_{t}-6}{\left(x_{t}-1\right)^{4}} \ln x_{t}\right] \\
& -\frac{s_{W}^{2}}{36}\left[\frac{x_{t}\left(18 x_{t}^{3}-139 x_{t}^{2}+274 x_{t}-129\right)}{2\left(x_{t}-1\right)^{3}}+\frac{24 x_{t}^{4}-33 x_{t}^{3}-45 x_{t}^{2}+50 x_{t}-8}{\left(x_{t}-1\right)^{4}} \ln x_{t}\right] .
\end{aligned}
$$

The GIM mechanism has eliminated those contributions which are independent of the virtual top-quark mass. However, the $\ln x_{t}$ terms in the Wilson coefficients $C_{S}^{\mathrm{SM}}$ and $C_{P}^{\mathrm{SM}}$ do not vanish in the massless limit: at $x_{t} \ll 1, C_{S}^{\mathrm{SM}} \sim-\frac{1}{12} \ln x_{t}$ and $C_{P}^{\mathrm{SM}} \sim$ $-\frac{1}{4}\left(1-\frac{8}{9} s_{W}^{2}\right) \ln x_{t}$. These infrared-sensitive terms arise from diagrams 1.1 and 2.1 in both gauges. The corresponding contributions from virtual up and charm quarks cancel in the matching process with the low-energy effective theory, which has the same infrared behaviour. $^{2}$

\subsection{Wilson coefficients in the A2HDM}

In the $\mathrm{A} 2 \mathrm{HDM}$, the only new contribution to $C_{10}$ comes from the $Z$-penguin diagrams shown in figure 5 . The result is gauge independent and given by

$$
C_{10}^{\mathrm{A} 2 \mathrm{HDM}}=C_{10}^{\mathrm{Z} \text { penguin, } \mathrm{A} 2 \mathrm{HDM}}=\left|\varsigma_{u}\right|^{2} \frac{x_{t}^{2}}{8}\left[\frac{1}{x_{H^{+}}-x_{t}}+\frac{x_{H^{+}}}{\left(x_{H^{+}}-x_{t}\right)^{2}}\left(\ln x_{t}-\ln x_{H^{+}}\right)\right] .
$$

In the particular case of the type-II $2 \mathrm{HDM}$ (or MSSM), $\varsigma_{u}=1 / \tan \beta$, this result agrees with the one calculated in ref. [36].

The box diagrams shown in figure 6 involve charged scalar exchanges and contribute to the Wilson coefficients $C_{S}^{\mathrm{A} 2 \mathrm{HDM}}$ and $C_{P}^{\mathrm{A} 2 \mathrm{HDM}}$. The contributions from diagrams 6.3 and 6.4

\footnotetext{
${ }^{2}$ In the low-energy effective theory the same $\ln x_{c}\left(\ln x_{u}\right)$ terms appear from analogous diagrams with a $c \bar{\nu}_{\ell}\left(u \bar{\nu}_{\ell}\right)$ or $c \bar{c}(u \bar{u})$ loop connecting two four-fermion operators.
} 

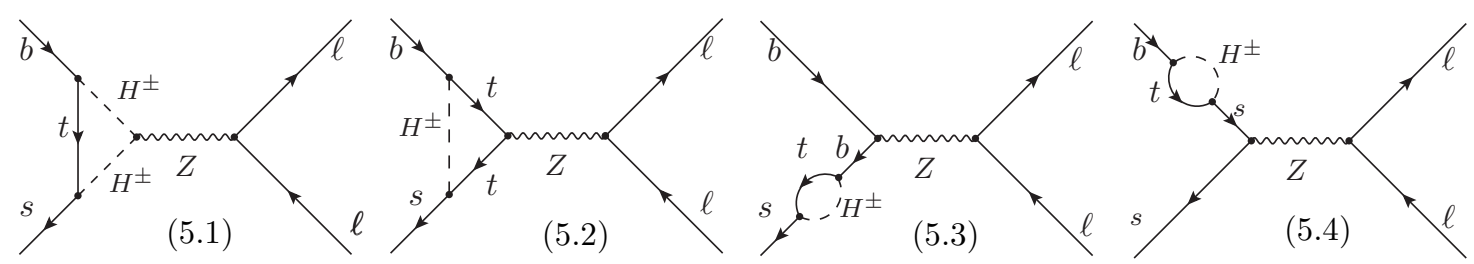

Figure 5. Z-penguin diagrams involving $H^{ \pm}$exchanges in the A2HDM.
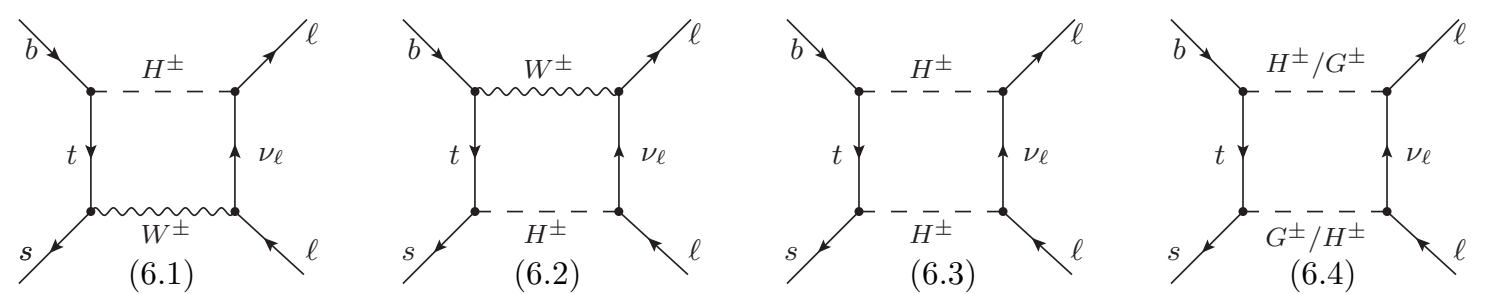

Figure 6. Box diagrams involving $H^{ \pm}$exchanges in the A2HDM. Diagrams with Goldstone bosons are absent in the unitary gauge.

can be neglected, since they are proportional to $m_{\ell}^{2} / M_{W}^{2}$. For the scalar coefficients we find the results:

$$
\begin{aligned}
C_{S, \text { Feynman }}^{\text {box, A2HDM }}= & \frac{x_{t}}{8\left(x_{H^{+}}-x_{t}\right)}\left\{\varsigma_{\ell} \varsigma_{u}^{*}\left[\frac{x_{t}-x_{H^{+}}}{\left(x_{H^{+}}-1\right)\left(x_{t}-1\right)}+\frac{x_{t}}{\left(x_{t}-1\right)^{2}} \ln x_{t}-\frac{x_{H^{+}}}{\left(x_{H^{+}}-1\right)^{2}} \ln x_{H^{+}}\right]\right. \\
& -\varsigma_{u} \varsigma_{\ell}^{*}\left[\frac{1}{x_{H^{+}}-1}+\frac{x_{H^{+}}}{\left(x_{H^{+}}-x_{t}\right)\left(x_{t}-1\right)} \ln x_{t}-\frac{x_{H^{+}}\left(2 x_{H^{+}}-x_{t}-1\right)}{\left(x_{H^{+}}-x_{t}\right)\left(x_{H^{+}}-1\right)^{2}} \ln x_{H^{+}}\right] \\
& \left.+2 \varsigma_{d} \varsigma_{\ell}^{*}\left[\frac{1}{x_{H^{+}}-1} \ln x_{H^{+}}-\frac{1}{x_{t}-1} \ln x_{t}\right]\right\}, \\
C_{S, \text { Unitary }}^{\text {box, A2HDM }}= & \frac{x_{t}}{8\left(x_{H^{+}}-x_{t}\right)}\left\{\varsigma_{\ell} \varsigma_{u}^{*}\left[\frac{x_{t}}{x_{t}-1} \ln x_{t}-\frac{x_{H^{+}}}{x_{H^{+}}-1} \ln x_{H^{+}}\right]\right. \\
& +\varsigma_{u} \varsigma_{\ell}^{*}\left[1-\frac{x_{H^{+}}-x_{t}^{2}}{\left(x_{H^{+}}-x_{t}\right)\left(x_{t}-1\right)} \ln x_{t}-\frac{x_{H^{+}}\left(x_{t}-1\right)}{\left(x_{H^{+}}-x_{t}\right)\left(x_{H^{+}}-1\right)} \ln x_{H^{+}}\right] \\
& \left.+2 \varsigma_{d} \varsigma_{\ell}^{*}\left[\ln x_{t}-\ln x_{H^{+}}\right]\right\},
\end{aligned}
$$

while the pseudoscalar contributions are given by:

$$
\begin{aligned}
& C_{P, \text { Feynman }}^{\text {box, A2HDM }}=-\left.C_{S, \text { Feynman }}^{\text {box, A2HDM }}\right|_{\varsigma_{\ell} \varsigma_{u}^{*} \rightarrow-\varsigma_{\ell} \varsigma_{u}^{*}}, \\
& C_{P, \text { Unitary }}^{\text {box, A2HDM }}=-\left.C_{S, \text { Unitary }}^{\text {box, A2HDM }}\right|_{\varsigma_{\ell} \varsigma_{u}^{*} \rightarrow-\varsigma_{\ell} \varsigma_{u}^{*}} .
\end{aligned}
$$

Most of the previous calculations in the literature focused on the type-II $2 \mathrm{HDM}$ in the large $\tan \beta$ limit; i.e., only those contributions proportional to $\tan ^{2} \beta$ were kept, which correspond to the $\varsigma_{d} \varsigma_{\ell}^{*}$ terms in eqs. (3.26)-(3.29). For this specific case, our results agree with ref. [28]. 

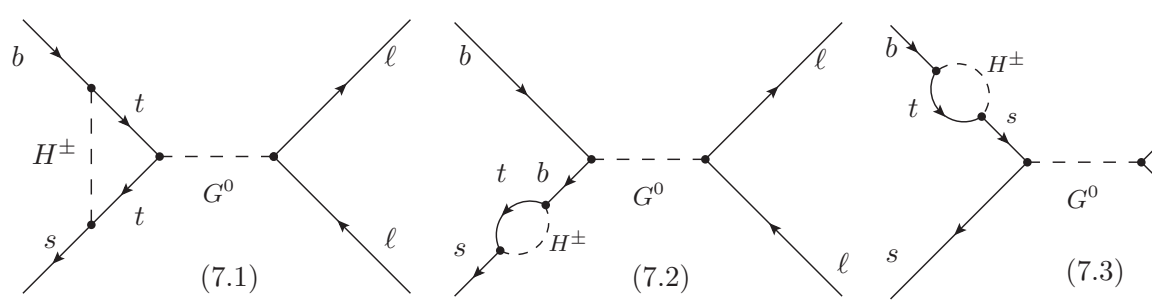

$(7.3)$

Figure 7. Goldstone-boson penguin diagrams involving $H^{ \pm}$exchanges in the A2HDM. These contributions are absent in the unitary gauge.

Similarly to the SM case, the coefficient $C_{P}^{\mathrm{A} 2 \mathrm{HDM}}$ receives additional contributions from $Z$ - and Goldstone-penguin diagrams shown in figures 5 and 7 , respectively. They are given by:

$$
\begin{aligned}
& C_{P, \text { Feynman }}^{\mathrm{Z} \text { penguin, A2HDM }}=\frac{x_{t}}{4\left(x_{H^{+}}-x_{t}\right)^{2}}\left\{\varsigma_{d} \varsigma_{u}^{*}\left[-\frac{x_{t}+x_{H^{+}}}{2}+\frac{x_{t} x_{H^{+}}}{x_{H^{+}}-x_{t}}\left(\ln x_{H^{+}}-\ln x_{t}\right)\right]\right. \\
& \left.+\left|\varsigma_{u}\right|^{2} \frac{1}{6\left(x_{H^{+}}-x_{t}\right)}\left[\frac{x_{H^{+}}^{2}-8 x_{H^{+}} x_{t}-17 x_{t}^{2}}{6}+\frac{x_{t}^{2}\left(3 x_{H^{+}}+x_{t}\right)}{x_{H^{+}}-x_{t}}\left(\ln x_{H^{+}}-\ln x_{t}\right)\right]\right\} \\
& +s_{W}^{2} \frac{x_{t}}{6\left(x_{H^{+}}-x_{t}\right)^{2}}\left\{\varsigma_{d} \varsigma_{u}^{*}\left[\frac{5 x_{t}-3 x_{H^{+}}}{2}+\frac{x_{H^{+}}\left(2 x_{H^{+}}-3 x_{t}\right)}{x_{H^{+}}-x_{t}}\left(\ln x_{H^{+}}-\ln x_{t}\right)\right]\right. \\
& -\left|\varsigma_{u}\right|^{2} \frac{1}{6\left(x_{H^{+}}-x_{t}\right)}\left[\frac{4 x_{H^{+}}^{3}-12 x_{H^{+}}^{2} x_{t}+9 x_{H^{+}} x_{t}^{2}+3 x_{t}^{3}}{x_{H^{+}}-x_{t}}\left(\ln x_{H^{+}}-\ln x_{t}\right)\right. \\
& \left.\left.-\frac{17 x_{H^{+}}^{2}-64 x_{H^{+}} x_{t}+71 x_{t}^{2}}{6}\right]\right\} \\
& C_{P, \text { Feynman }}^{\mathrm{GB} \text { penguin, } \mathrm{A} 2 \mathrm{HDM}}=\left|\varsigma_{u}\right|^{2}\left(1-s_{W}^{2}\right) \frac{x_{t}^{2}}{4\left(x_{H^{+}}-x_{t}\right)^{2}}\left[x_{H^{+}}\left(\ln x_{H^{+}}-\ln x_{t}\right)+x_{t}-x_{H^{+}}\right] \text {. }
\end{aligned}
$$

The gauge dependence of these two contributions compensates each other. Since there is no contribution from Goldstone-penguin topologies in the unitary gauge, the $Z$-penguin result should satisfy in this case:

$$
C_{P, \text { Unitay }}^{\mathrm{Z} \text { penguin, } \mathrm{A} 2 \mathrm{HDM}}=C_{P, \text { Feynman }}^{\mathrm{Z} \text { penguin, } \mathrm{A} 2 \mathrm{HDM}}+C_{P, \text { Feynman }}^{\mathrm{GB} \text { penguin, } \mathrm{A} 2 \mathrm{HDM}} .
$$

This relation has been validated by the actual calculation.

\subsubsection{Neutral scalar exchange}

The Wilson coefficients $C_{S}^{\mathrm{A} 2 \mathrm{HDM}}$ and $C_{P}^{\mathrm{A} 2 \mathrm{HDM}}$ receive a direct tree-level contribution from the scalar-exchange diagram shown in figure 8 , where the FCNC vertex $\varphi_{i}^{0} \bar{s} b$ is generated by the local operator in eq. (2.15). This contribution must be combined together with the scalar penguin diagrams shown in figure 9 . The structure of the common $\varphi_{i}^{0} \bar{\ell} \ell$ vertex relates the resulting scalar and pseudoscalar Wilson coefficients, which take the form:

$$
C_{S}^{\varphi_{i}^{0}, \mathrm{~A} 2 \mathrm{HDM}}=\sum_{\varphi_{i}^{0}} \operatorname{Re}\left(y_{\ell}^{\varphi_{i}^{0}}\right) \hat{C}^{\varphi_{i}^{0}}, \quad C_{P}^{\varphi_{i}^{0}, \mathrm{~A} 2 \mathrm{HDM}}=i \sum_{\varphi_{i}^{0}} \operatorname{Im}\left(y_{\ell}^{\varphi_{i}^{0}}\right) \hat{C}^{\varphi_{i}^{0}}
$$




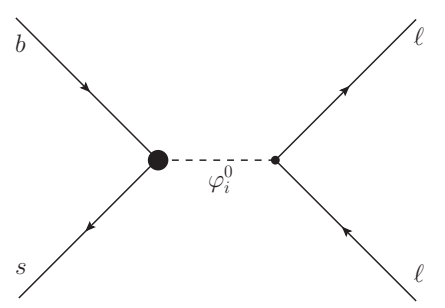

Figure 8. Tree-level FCNC diagram mediated by the neutral scalars $\varphi_{i}^{0}=\{h, H, A\}$.

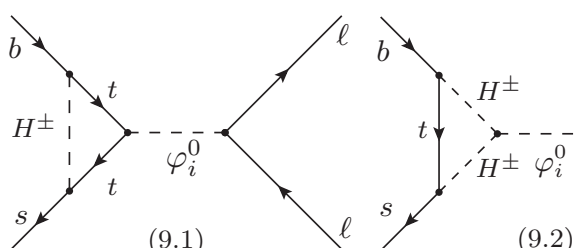

$(9.1)$

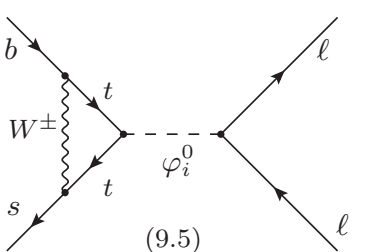

$(9.5)$

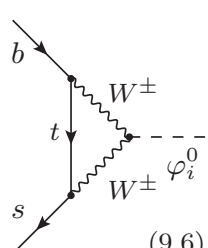

$9.2)$
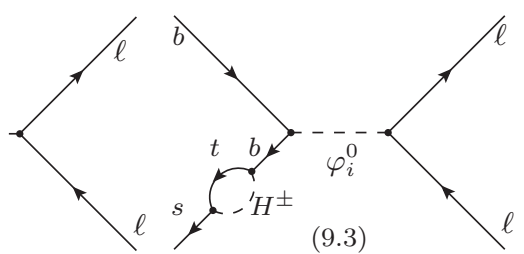

$(9.3)$

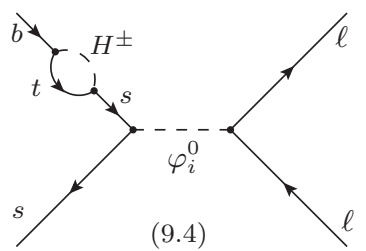

$(9.4)$
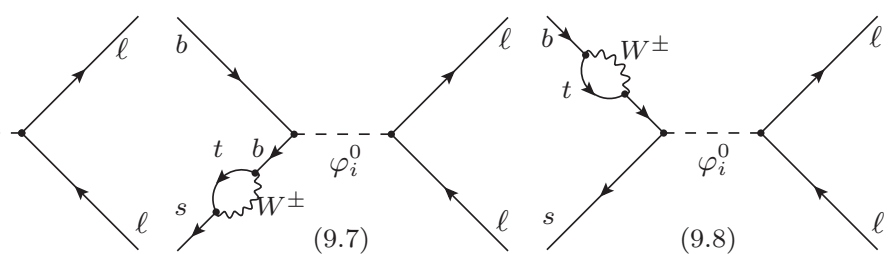

$(9.8)$
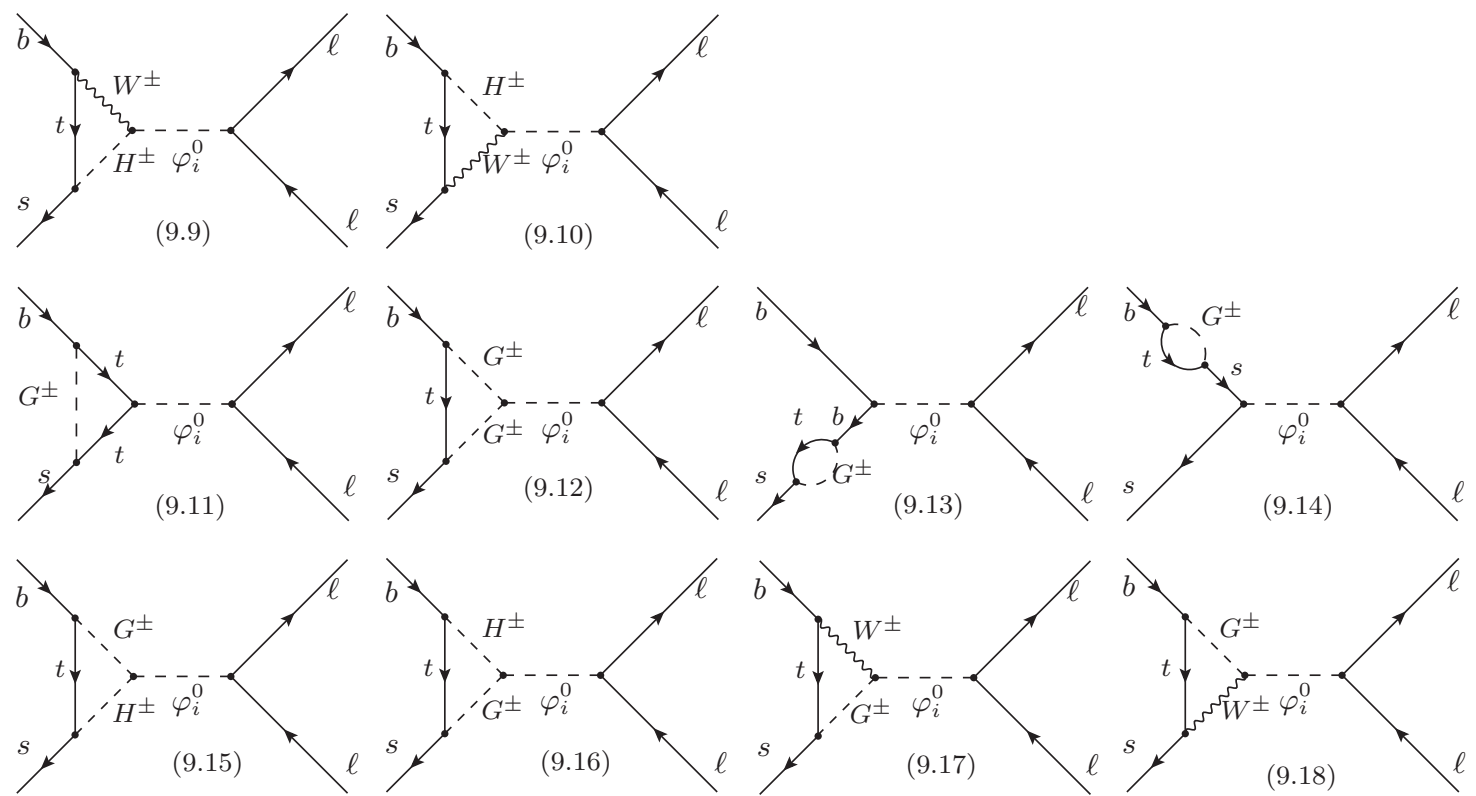

Figure 9. Scalar penguin diagrams in the A2HDM, where $\varphi_{i}^{0}=\{h, H, A\}$. Diagrams 9.11 to 9.18 are absent in the unitary gauge.

The contributions from diagrams 9.4, 9.7, 9.8 and 9.14 are proportional to the light-quark mass $m_{q}$ and, therefore, vanish in our massless approximation. Diagrams 9.1, 9.3, 9.11 and 9.13 in Feynman gauge and diagrams 9.1, 9.3, 9.5, 9.6, 9.9 and 9.10 in unitary gauge generate a divergent contribution, which is not eliminated by the GIM 
mechanism; i.e., it remains even after summing over contributions of the three virtual uptype quarks. This divergence matches exactly the expected behaviour predicted through the RGEs, which originated in the local term $\mathcal{L}_{\mathrm{FCNC}}$. Thus, the one-loop divergence is cancelled by the renormalization of the coupling $\mathcal{C}$ in eq. (2.16) which, moreover, reabsorbs the $\mu$ dependence of the loops into the combination $\mathcal{C}_{R}\left(M_{W}\right)=\mathcal{C}_{R}(\mu)-\ln \left(M_{W} / \mu\right)$.

The scalar penguin diagrams 9.2, 9.12, 9.15 and 9.16 involve the cubic couplings $\varphi_{i}^{0} H^{+} H^{-}, \varphi_{i}^{0} G^{+} G^{-}, \varphi_{i}^{0} H^{+} G^{-}$and $\varphi_{i}^{0} G^{+} H^{-}$, respectively, which are functions of the scalar-potential parameters. Since the last three couplings can be fully determined in terms of the vacuum expectation value $v$ and the scalar masses and mixings, we can express the total scalar-exchange (tree-level plus one-loop penguin) contribution in the form:

$$
\begin{aligned}
\hat{C}^{\varphi_{i}^{0}}=x_{t}\{ & \frac{1}{2 x_{\varphi_{i}^{0}}}\left(\varsigma_{u}-\varsigma_{d}\right)\left(1+\varsigma_{u}^{*} \varsigma_{d}\right)\left(\mathcal{R}_{i 2}+i \mathcal{R}_{i 3}\right) \mathcal{C}_{R}\left(M_{W}\right)+\frac{v^{2}}{M_{\varphi_{i}^{0}}^{2}} \lambda_{H^{+} H^{-}}^{\varphi^{0}} g_{0}\left(x_{t}, x_{H^{+}}, \varsigma_{u}, \varsigma_{d}\right) \\
& \left.+\sum_{j=1}^{3} \mathcal{R}_{i j} \xi_{j}\left[\frac{1}{2 x_{\varphi_{i}^{0}}} g_{j}^{(a)}\left(x_{t}, x_{H^{+}}, \varsigma_{u}, \varsigma_{d}\right)+g_{j}^{(b)}\left(x_{t}, x_{H^{+}}, \varsigma_{u}, \varsigma_{d}\right)\right]\right\}
\end{aligned}
$$

where $\lambda_{H^{+} H^{-}}^{\varphi^{0}}=\lambda_{3} \mathcal{R}_{i 1}+\lambda_{7}^{R} \mathcal{R}_{i 2}-\lambda_{7}^{I} \mathcal{R}_{i 3}, \xi_{1}=\xi_{2}=1$ and $\xi_{3}=i$. The functions $g_{0}\left(x_{t}, x_{H^{+}}, \varsigma_{u}, \varsigma_{d}\right), g_{j}^{(a)}\left(x_{t}, x_{H^{+}}, \varsigma_{u}, \varsigma_{d}\right)$ and $g_{j}^{(b)}\left(x_{t}, x_{H^{+}}, \varsigma_{u}, \varsigma_{d}\right)$ are given in the appendix, both in the Feynman and unitary gauges, together with the separate contributions from each diagram in figure 9 . In the limit $\varsigma_{u, d} \rightarrow 0, x_{H, A} \rightarrow \infty, x_{h} \rightarrow x_{h_{\mathrm{SM}}}, \mathcal{R}_{i 2, i 3} \rightarrow 0$, $\mathcal{R}_{11} \rightarrow 1$, this result reduces to the SM expression in eqs. (3.16) and (3.17).

The orthogonality relation $[20,21]$

$$
\sum_{i=1}^{3} y_{\ell}^{\varphi_{i}^{0}} \mathcal{R}_{i j}=\delta_{j 1}+\left(\delta_{j 2}+i \delta_{j 3}\right) \varsigma_{\ell}
$$

allows us to separate the total contribution from the functions $g_{j}^{(b)}\left(x_{t}, x_{H^{+}}, \varsigma_{u}, \varsigma_{d}\right)$, which does not depend on the neutral scalar masses:

$$
\begin{aligned}
& \left.C_{S}^{\varphi_{i}^{0}, \mathrm{~A} 2 \mathrm{HDM}}\right|_{g^{(b)}}=x_{t}\left[g_{1}^{(b)}+\operatorname{Re}\left(\varsigma_{\ell}\right) g_{2}^{(b)}-i \operatorname{Im}\left(\varsigma_{\ell}\right) g_{3}^{(b)}\right] \\
& C_{P}^{\varphi_{i}^{0},\left.\mathrm{~A} 2 \mathrm{HDM}\right|_{g^{(b)}}}=x_{t}\left[i \operatorname{Im}\left(\varsigma_{\ell}\right) g_{2}^{(b)}-\operatorname{Re}\left(\varsigma_{\ell}\right) g_{3}^{(b)}\right] .
\end{aligned}
$$

It is also noted that the functions $g_{j}^{(b)}\left(x_{t}, x_{H^{+}}, \varsigma_{u}, \varsigma_{d}\right)$ only receive contributions in the Feynman gauge, because they arise from the scalar penguin diagrams involving the Goldstone bosons. Actually, the gauge dependent pieces from the box diagrams shown in figures 1 and 6 are exactly cancelled by these terms:

$$
\begin{aligned}
C_{S, \text { Unitary }}^{\text {box, SM }}-C_{S, \text { Feynman }}^{\text {box, SM }} & =x_{t} g_{1}^{(b)}, \\
C_{S, \text { Unitary }}^{\text {box, A2HDM }}-C_{S, \text { Feynman }}^{\text {box, A2HDM }} & =x_{t}\left[\operatorname{Re}\left(\varsigma_{\ell}\right) g_{2}^{(b)}-i \operatorname{Im}\left(\varsigma_{\ell}\right) g_{3}^{(b)}\right], \\
C_{P, \text { Unitary }}^{\text {box, A2HDM }}-C_{P, \text { Feynman }}^{\text {box, A2HDM }} & =x_{t}\left[i \operatorname{Im}\left(\varsigma_{\ell}\right) g_{2}^{(b)}-\operatorname{Re}\left(\varsigma_{\ell}\right) g_{3}^{(b)}\right] .
\end{aligned}
$$


The remaining contributions in eq. (3.34), which are all proportional to $1 / M_{\varphi_{i}^{0}}^{2}$, are gauge independent but are sensitive to the scalar mixing parameters. Nevertheless, a naive mixing-independent estimate can be obtained in the limit of degenerate neutral-scalar masses:

$$
\begin{aligned}
C_{S}^{\varphi_{i}^{0}}, \mathrm{~A} 2 \mathrm{HDM}||_{\mathcal{C}+g_{0}+g^{(a)}}^{x_{h}=x_{H}=x_{A}}= & \frac{x_{t}}{2 x_{h}}\left\{\left(\varsigma_{u}-\varsigma_{d}\right)\left(1+\varsigma_{u}^{*} \varsigma_{d}\right) \mathcal{C}_{R}\left(M_{W}\right)\left[\operatorname{Re}\left(\varsigma_{\ell}\right)-i \operatorname{Im}\left(\varsigma_{\ell}\right)\right]\right. \\
& +\frac{2 v^{2}}{M_{W}^{2}} g_{0}\left[\lambda_{3}+\lambda_{7}^{R} \operatorname{Re}\left(\varsigma_{\ell}\right)+\lambda_{7}^{I} \operatorname{Im}\left(\varsigma_{\ell}\right)\right] \\
& \left.+g_{1}^{(a)}+\operatorname{Re}\left(\varsigma_{\ell}\right) g_{2}^{(a)}-i \operatorname{Im}\left(\varsigma_{\ell}\right) g_{3}^{(a)}\right\} \\
C_{P}^{\varphi_{i}^{0}},\left.\mathrm{~A} 2 \mathrm{HDM}\right|_{\mathcal{C}+g_{0}+g^{(a)}} ^{x_{h}=x_{H}=x_{A}}= & \frac{x_{t}}{2 x_{h}}\left\{\left(\varsigma_{u}-\varsigma_{d}\right)\left(1+\varsigma_{u}^{*} \varsigma_{d}\right) \mathcal{C}_{R}\left(M_{W}\right)\left[i \operatorname{Im}\left(\varsigma_{\ell}\right)-\operatorname{Re}\left(\varsigma_{\ell}\right)\right]\right. \\
& +\frac{2 v^{2}}{M_{W}^{2}} g_{0} i\left[\lambda_{7}^{R} \operatorname{Im}\left(\varsigma_{\ell}\right)-\lambda_{7}^{I} \operatorname{Re}\left(\varsigma_{\ell}\right)\right] \\
& \left.+i \operatorname{Im}\left(\varsigma_{\ell}\right) g_{2}^{(a)}-\operatorname{Re}\left(\varsigma_{\ell}\right) g_{3}^{(a)}\right\}
\end{aligned}
$$

We shall perform our phenomenological analyses in the CP-conserving limit, with real potential and alignment parameters, where $A=S_{3}$ is a CP-odd state while $H$ and $h$ are two CP-even states defined by the rotation in eq. (2.6). The $1 / x_{\varphi_{i}^{0}}$ contributions take then the form:

$$
\begin{aligned}
& \left.C_{S}^{\varphi_{i}^{0}, \mathrm{~A} 2 \mathrm{HDM}}\right|_{\mathcal{C}+g_{0}+g^{(a)}} ^{\mathrm{CP} \text { con. }}=\frac{x_{t}}{2 x_{h}}\left(c_{\tilde{\alpha}}+s_{\tilde{\alpha}} \varsigma_{\ell}\right)\left\{s_{\tilde{\alpha}}\left(\varsigma_{u}-\varsigma_{d}\right)\left(1+\varsigma_{u} \varsigma_{d}\right) \mathcal{C}_{R}\left(M_{W}\right)\right. \\
& \left.+\left(c_{\tilde{\alpha}} \lambda_{3}+s_{\tilde{\alpha}} \lambda_{7}\right) \frac{2 v^{2}}{M_{W}^{2}} g_{0}+c_{\tilde{\alpha}} g_{1}^{(a)}+s_{\tilde{\alpha}} g_{2}^{(a)}\right\} \\
& +\frac{x_{t}}{2 x_{H}}\left(c_{\tilde{\alpha}} \varsigma_{\ell}-s_{\tilde{\alpha}}\right)\left\{c_{\tilde{\alpha}}\left(\varsigma_{u}-\varsigma_{d}\right)\left(1+\varsigma_{u} \varsigma_{d}\right) \mathcal{C}_{R}\left(M_{W}\right)\right. \\
& \left.-\left(s_{\tilde{\alpha}} \lambda_{3}-c_{\tilde{\alpha}} \lambda_{7}\right) \frac{2 v^{2}}{M_{W}^{2}} g_{0}-s_{\tilde{\alpha}} g_{1}^{(a)}+c_{\tilde{\alpha}} g_{2}^{(a)}\right\}, \\
& \left.C_{P}^{\varphi_{i}^{0}, \mathrm{~A} 2 \mathrm{HDM}}\right|_{\mathcal{C}+g_{0}+g^{(a)}} ^{\mathrm{CP} \text { con. }}=-\varsigma_{\ell} \frac{x_{t}}{2 x_{A}}\left[\left(\varsigma_{u}-\varsigma_{d}\right)\left(1+\varsigma_{u} \varsigma_{d}\right) \mathcal{C}_{R}\left(M_{W}\right)+g_{3}^{(a)}\right],
\end{aligned}
$$

where $c_{\tilde{\alpha}}=\cos \tilde{\alpha}$ and $s_{\tilde{\alpha}}=\sin \tilde{\alpha}$. For degenerate neutral scalars, this reproduces the results in eqs. (3.41) and (3.42) (in the CP-conserving limit).

The terms proportional to $C_{R}\left(M_{W}\right)$ in eqs. (3.43) and (3.44) are absent in $\mathcal{Z}_{2^{-}}$ symmetric models, because the alignment conditions are protected by the $\mathcal{Z}_{2}$ symmetry at any scale. In the particular case of the type-II $2 \mathrm{HDM}$ at large $\tan \beta$, the only terms enhanced by a factor $\tan ^{2} \beta$ originate from the $\varsigma_{\ell} g_{2}^{(a)}$ (for $C_{S}$ ) and $\varsigma_{\ell} g_{3}^{(a)}$ (for $C_{P}$ ) contributions, due to the factors $\varsigma_{d}^{2} \varsigma_{u}^{*}$ and $\varsigma_{d}$ in the definitions for $g_{2}^{(a)}$ and $g_{3}^{(a)}$ (see eqs. (A.29) and (A.30)). In this specific case, our results agree with the ones calculated in ref. [28]. Especially, we confirmed the observation that the dependence on the masses of the neutral Higgs bosons from the penguin and fermion self-energy diagrams drops out in their sum without invoking any relation between the mixing angle and the Higgs masses [28]. 


\section{$3.5 \quad B_{s, d}^{0} \rightarrow \ell^{+} \ell^{-}$branching ratio}

Due to the pseudoscalar nature of the $B_{q}$ meson, only the following two hadronic matrix elements are involved in $B_{s, d}^{0} \rightarrow \ell^{+} \ell^{-}$decays:

$$
\begin{aligned}
\left\langle 0\left|\bar{q} \gamma_{\mu} \gamma_{5} b\right| \bar{B}_{q}(p)\right\rangle & =i f_{B_{q}} p_{\mu}, \\
\left\langle 0\left|\bar{q} \gamma_{5} b\right| \bar{B}_{q}(p)\right\rangle & =-i f_{B_{q}} \frac{M_{B_{q}}^{2}}{m_{b}+m_{q}},
\end{aligned}
$$

where $f_{B_{q}}$ and $M_{B_{q}}$ are the $B_{q}$-meson decay constant and mass, respectively. The second equation follows from the first one by using the QCD equation of motion for the quark fields.

Starting with eq. (3.1) and using eq. (3.45), we can express the branching ratio of $B_{s, d}^{0} \rightarrow \ell^{+} \ell^{-}$decays as

$$
\begin{aligned}
\mathcal{B}\left(B_{q}^{0} \rightarrow \ell^{+} \ell^{-}\right) & =\frac{\tau_{B_{q}} G_{F}^{4} M_{W}^{4}}{8 \pi^{5}}\left|V_{t b} V_{t q}^{*} C_{10}^{\mathrm{SM}}\right|^{2} f_{B_{q}}^{2} M_{B_{q}} m_{\ell}^{2} \sqrt{1-\frac{4 m_{\ell}^{2}}{M_{B_{q}}^{2}}}\left[|P|^{2}+|S|^{2}\right] \\
& =\mathcal{B}\left(B_{q}^{0} \rightarrow \ell^{+} \ell^{-}\right)_{\mathrm{SM}}\left[|P|^{2}+|S|^{2}\right]
\end{aligned}
$$

where $\tau_{B_{q}}$ is the $B_{q^{-}}$-meson mean lifetime, and $P$ and $S$ are defined, respectively, as [71-73]

$$
\begin{aligned}
P & \equiv \frac{C_{10}}{C_{10}^{\mathrm{SM}}}+\frac{M_{B_{q}}^{2}}{2 M_{W}^{2}}\left(\frac{m_{b}}{m_{b}+m_{q}}\right) \frac{C_{P}-C_{P}^{\mathrm{SM}}}{C_{10}^{\mathrm{SM}}} \equiv|P| e^{i \phi_{P}}, \\
S & \equiv \sqrt{1-\frac{4 m_{\ell}^{2}}{M_{B_{q}}^{2}}} \frac{M_{B_{q}}^{2}}{2 M_{W}^{2}}\left(\frac{m_{b}}{m_{b}+m_{q}}\right) \frac{C_{S}-C_{S}^{\mathrm{SM}}}{C_{10}^{\mathrm{SM}}} \equiv|S| e^{i \phi_{S}} .
\end{aligned}
$$

We have approximated the negligibly small (and usually neglected) SM scalar/pseudoscalar contributions ${ }^{3} C_{S}^{\mathrm{SM}}$ and $C_{P}^{\mathrm{SM}}$ to first order in $M_{B_{q}}^{2} / M_{W}^{2}$. In the SM, $P=1$ and $S=0$. In a generic case, however, $P$ and $S$ can carry nontrivial CP-violating phases $\phi_{P}$ and $\phi_{S}$. It is also noted that, even in models with comparable Wilson coefficients, the contributions from $\mathcal{O}_{S}$ and $\mathcal{O}_{P}$ are suppressed by a factor $M_{B_{q}}^{2} / M_{W}^{2}$ with respect to that from $\mathcal{O}_{10}$. Therefore, unless there were large enhancements for $C_{S}$ and $C_{P}$, the coefficient $C_{10}$ still provides the dominant contribution to the branching ratio.

In order to compare with the experimental measurement, the effect of $B_{q}^{0}-\bar{B}_{q}^{0}$ oscillations should be taken into account, and the resulting averaged time-integrated branching ratio is given by [71-73]

$$
\overline{\mathcal{B}}\left(B_{q}^{0} \rightarrow \ell^{+} \ell^{-}\right)=\left[\frac{1+\mathcal{A}_{\Delta \Gamma}^{\ell \ell} y_{q}}{1-y_{q}^{2}}\right] \mathcal{B}\left(B_{q}^{0} \rightarrow \ell^{+} \ell^{-}\right),
$$

where $\mathcal{A}_{\Delta \Gamma}^{\ell \ell}$ is a time-dependent observable introduced firstly in ref. [72, 73], and $y_{q}$ is related to the decay width difference $\Delta \Gamma_{q}$ between the two $B_{q}$-meson mass eigenstates,

$$
y_{q} \equiv \frac{\Gamma_{L}^{q}-\Gamma_{H}^{q}}{\Gamma_{L}^{q}+\Gamma_{H}^{q}}=\frac{\Delta \Gamma_{q}}{2 \Gamma_{q}},
$$

\footnotetext{
${ }^{3}$ Here, $C_{S}^{\mathrm{SM}}$ and $C_{P}^{\mathrm{SM}}$ denote the full SM contribution, including the Higgs-penguin terms.
} 
with $\Gamma_{H(L)}^{q}$ denoting the heavier (lighter) eigenstate decay width and $\Gamma_{q}=\tau_{B_{q}}^{-1}$ the average $B_{q}$-meson width. Within the SM, $\mathcal{A}_{\Delta \Gamma}^{\ell \ell}=1$ and the averaged time-integrated branching ratio is given by

$$
\begin{aligned}
\overline{\mathcal{B}}\left(B_{q}^{0} \rightarrow \ell^{+} \ell^{-}\right)_{\mathrm{SM}} & =\frac{1}{1-y_{q}} \mathcal{B}\left(B_{q}^{0} \rightarrow \ell^{+} \ell^{-}\right)_{\mathrm{SM}} \\
& =\frac{G_{F}^{4} M_{W}^{4}}{8 \pi^{5} \Gamma_{H}^{q}}\left|V_{t b} V_{t q}^{*} C_{10}^{\mathrm{SM}}\right|^{2} f_{B_{q}}^{2} M_{B_{q}} m_{\ell}^{2} \sqrt{1-\frac{4 m_{\ell}^{2}}{M_{B_{q}}^{2}}}
\end{aligned}
$$

By exploiting eqs. (3.46) and (3.51), we can rewrite eq. (3.49) as

$$
\begin{aligned}
\overline{\mathcal{B}}\left(B_{q}^{0} \rightarrow \ell^{+} \ell^{-}\right) & =\left[\frac{1+\mathcal{A}_{\Delta \Gamma}^{\ell \ell} y_{q}}{1+y_{q}}\right]\left[|P|^{2}+|S|^{2}\right] \overline{\mathcal{B}}\left(B_{q}^{0} \rightarrow \ell^{+} \ell^{-}\right)_{\mathrm{SM}} \\
& \doteq \overline{\mathcal{B}}\left(B_{q}^{0} \rightarrow \ell^{+} \ell^{-}\right)_{\mathrm{SM}}\left[|P|^{2}+\left(1-\frac{\Delta \Gamma_{q}}{\Gamma_{L}^{q}}\right)|S|^{2}\right]
\end{aligned}
$$

where the second line is valid only in the absence of beyond-SM sources of CP violation, which will be assumed in the following. ${ }^{4}$

\section{Numerical results}

\subsection{Input parameters}

To evaluate numerically the branching ratios in eqs. (3.51) and (3.52), we need several input parameters collected in table 2. For the matching scale $\mu_{0} \sim \mathcal{O}\left(M_{W}\right)$ and the low-energy scale $\mu_{b} \sim \mathcal{O}\left(m_{b}\right)$, we fix them to $\mu_{0}=160 \mathrm{GeV}$ and $\mu_{b}=5 \mathrm{GeV}$ [55]. In addition, the on-shell scheme is adopted for the EW parameters, which means that the $Z$ boson and top-quark masses coincide with their pole masses, and the weak angle is given by $s_{W}^{2} \equiv 1-M_{W}^{2} / M_{Z}^{2}$, where $M_{W}=80.359 \pm 0.012 \mathrm{GeV}$ is the $W$-boson on-shell mass obtained according to the fit formulae in eqs. (6) and (9) of ref. [101].

For the top-quark mass, we assume that the combined measurement of Tevatron and LHC [95] corresponds to the pole mass, but increase its systematic error by $1 \mathrm{GeV}$ to account for the intrinsic ambiguity in the $m_{t}$ definition; i.e. we shall take $M_{t}=(173.34 \pm$ $0.27 \pm 1.71) \mathrm{GeV}$. With the aid of the Mathematica package RunDec [102], four-loop QCD RGEs are applied to evolve the strong coupling $\alpha_{s}(\mu)$ as well as the $\overline{\mathrm{MS}}$ renormalized masses $m_{t}(\mu)$ and $m_{b, s}(\mu)$ between different scales, and a three-loop relation has been used to convert the pole mass $M_{t}$ to the scale-invariant mass $m_{t}\left(m_{t}\right)$, which gives $m_{t}\left(m_{t}\right) \simeq$ $163.30 \mathrm{GeV}$.

The decay constants $f_{B_{q}}$ are taken from the updated FLAG [96] average of $N_{f}=2+1$ lattice determinations, which are consistent with the naive weighted average of $N_{f}=$ $2+1[103-105]$ and $N_{f}=2+1+1[106,107]$ results. For the $B_{q}$-meson lifetimes, while a sizable decay width difference $\Delta \Gamma_{s}$ has been established [97], the approximation $1 / \Gamma_{H}^{d} \simeq$ $1 / \Gamma_{L}^{d} \equiv \tau_{B_{d}}$ can be safely set, given the tiny SM expectation for $\Delta \Gamma_{d} / \Gamma_{d}[108]$.

\footnotetext{
${ }^{4}$ The explicit formulae in a generic case with new CP-violating phases could be found in refs. [57, 71-73].
} 


\begin{tabular}{|ll|lr|}
\hline$G_{F}=1.1663787 \times 10^{-5} \mathrm{GeV}^{-2}$ & {$[94]$} & $f_{B_{s}}=227.7 \pm 4.5 \mathrm{MeV}$ & {$[96]$} \\
$\alpha_{s}\left(M_{Z}\right)=0.1185 \pm 0.0006$ & {$[94]$} & $f_{B_{d}}=190.5 \pm 4.2 \mathrm{MeV}$ & {$[96]$} \\
$\Delta \alpha_{\text {hadr }}\left(M_{Z}\right)=0.02772 \pm 0.00010$ & {$[94]$} & $\tau_{B_{s}}=1.516 \pm 0.011 \mathrm{ps}$ & {$[97]$} \\
$M_{Z}=91.1876 \pm 0.0021 \mathrm{GeV}$ & {$[94]$} & $\tau_{B_{d}}=1.519 \pm 0.007 \mathrm{ps}$ & {$[97]$} \\
$M_{t}=173.34 \pm 0.27 \pm 1.71 \mathrm{GeV}$ & {$[95]$} & $1 / \Gamma_{H}^{s}=1.615 \pm 0.021 \mathrm{ps}$ & {$[97]$} \\
$M_{h_{\mathrm{SM}}}=125.9 \pm 0.4 \mathrm{GeV}$ & {$[94]$} & $1 / \Gamma_{L}^{s}=1.428 \pm 0.013 \mathrm{ps}$ & {$[97]$} \\
$M_{B_{s}}=5366.77 \pm 0.24 \mathrm{MeV}$ & {$[94]$} & $\Delta \Gamma_{s}=0.081 \pm 0.011 \mathrm{ps}^{-1}$ & {$[97]$} \\
$M_{B_{d}}=5279.58 \pm 0.17 \mathrm{MeV}$ & {$[94]$} & $\left|V_{c b}\right|=(42.42 \pm 0.86) \times 10^{-3}$ & {$[98]$} \\
$m_{b}\left(m_{b}\right)=4.18 \pm 0.03 \mathrm{GeV}$ & {$[94]$} & $\left|V_{t b}^{*} V_{t s} / V_{c b}\right|=0.980 \pm 0.001$ & {$[99,100]$} \\
$m_{s}(2 \mathrm{GeV})=95 \pm 5 \mathrm{MeV}$ & {$[94]$} & $\left|V_{t b}^{*} V_{t d}\right|=0.0088 \pm 0.0003$ & {$[99,100]$} \\
$m_{\mu}=105.65837 \mathrm{MeV}$ & {$[94]$} & & \\
\hline
\end{tabular}

Table 2. Relevant input parameters used in our numerical analysis.

For the CKM matrix element $\left|V_{c b}\right|$, we adopt the recent inclusive fit performed by taking into account both the semileptonic data and the precise quark mass determinations from flavour-conserving processes [98]. However, one should be aware of the present disagreement between inclusive and exclusive determinations [96]. With $\left|V_{c b}\right|$ fixed in this way, the needed CKM factors are then obtained (within the SM) from the accurately known ratio $\left|V_{t b}^{*} V_{t s} / V_{c b}\right|[99,100]$.

\subsection{SM predictions}

Within the SM, only the Wilson coefficient $C_{10}^{\mathrm{SM}}$ is relevant and, using the fitting formula in eq. (4) of ref. [55] (which has been transformed to our convention for the effective Hamiltonian),

$$
\begin{aligned}
C_{10}^{\mathrm{SM}} & =-0.9604\left[\frac{M_{t}}{173.1 \mathrm{GeV}}\right]^{1.52}\left[\frac{\alpha_{s}\left(M_{Z}\right)}{0.1184}\right]^{-0.09}+0.0224\left[\frac{M_{t}}{173.1 \mathrm{GeV}}\right]^{0.89}\left[\frac{\alpha_{s}\left(M_{Z}\right)}{0.1184}\right]^{-0.09} \\
& =-0.9380\left[\frac{M_{t}}{173.1 \mathrm{GeV}}\right]^{1.53}\left[\frac{\alpha_{s}\left(M_{Z}\right)}{0.1184}\right]^{-0.09}
\end{aligned}
$$

The EW and QCD factors introduced in eq. (3.12) are extracted as:

$$
\eta_{Y}^{\mathrm{EW}}=0.977, \quad \eta_{Y}^{\mathrm{QCD}}=1.010 .
$$

With the input parameters collected in table 2, the SM predictions for the branching ratios of $B_{s, d}^{0} \rightarrow \ell^{+} \ell^{-}$decays are:

$$
\begin{aligned}
\overline{\mathcal{B}}\left(B_{s} \rightarrow e^{+} e^{-}\right) & =(8.58 \pm 0.59) \times 10^{-14}, \\
\overline{\mathcal{B}}\left(B_{s} \rightarrow \mu^{+} \mu^{-}\right) & =(3.67 \pm 0.25) \times 10^{-9}, \\
\overline{\mathcal{B}}\left(B_{s} \rightarrow \tau^{+} \tau^{-}\right) & =(7.77 \pm 0.53) \times 10^{-7} \\
\overline{\mathcal{B}}\left(B_{d} \rightarrow e^{+} e^{-}\right) & =(2.49 \pm 0.22) \times 10^{-15} \\
\overline{\mathcal{B}}\left(B_{d} \rightarrow \mu^{+} \mu^{-}\right) & =(1.06 \pm 0.10) \times 10^{-10} \\
\overline{\mathcal{B}}\left(B_{d} \rightarrow \tau^{+} \tau^{-}\right) & =(2.23 \pm 0.20) \times 10^{-8}
\end{aligned}
$$


where a $1.5 \%$ nonparametric uncertainty has been set to the branching ratios, and the main parametric uncertainties come from $f_{B_{q}}$ and the CKM matrix elements [55]. The small differences with respect to the results given in ref. [55] are due to our slightly different (more conservative) input value for the top-quark mass $M_{t}$.

In order to explore constraints on the model parameters, it is convenient to introduce the ratio [71-73]

$$
\bar{R}_{q \ell} \equiv \frac{\overline{\mathcal{B}}\left(B_{q}^{0} \rightarrow \ell^{+} \ell^{-}\right)}{\overline{\mathcal{B}}\left(B_{q}^{0} \rightarrow \ell^{+} \ell^{-}\right)_{\mathrm{SM}}}=\left[|P|^{2}+\left(1-\frac{\Delta \Gamma_{q}}{\Gamma_{L}^{q}}\right)|S|^{2}\right],
$$

where the hadronic factors and CKM matrix elements cancel out. Combining the theoretical SM predictions in eq. (4.3) with the experimental results in eqs. (1.2) and (1.4), we get

$$
\bar{R}_{s \mu}=0.79 \pm 0.20, \quad \bar{R}_{d \mu}=3.38_{-1.35}^{+1.53},
$$

to be compared with the SM expectation $\bar{R}_{s \mu}^{\mathrm{SM}}=\bar{R}_{d \mu}^{\mathrm{SM}}=1$.

Since only the $B_{s} \rightarrow \mu^{+} \mu^{-}$branching ratio is currently measured with a signal significance of $\sim 4.0 \sigma$ [54], we shall investigate the allowed parameter space of the A2HDM under the constraint from $\bar{R}_{s \mu}$ given in eq. (4.5). Although the experimental uncertainty is still quite large, it has already started to put stringent constraints on many models beyond the SM [71].

Notice that, in addition to modifying the ratios $\bar{R}_{q \ell}$, the scalar contributions to $B_{q}^{0}-\bar{B}_{q}^{0}$ mixings also change the fitted values of the relevant CKM parameters and, therefore, the normalization $\overline{\mathcal{B}}\left(B_{q}^{0} \rightarrow \ell^{+} \ell^{-}\right)_{\mathrm{SM}}$. This should be taken into account, once more precise $B_{q}^{0} \rightarrow \ell^{+} \ell^{-}$data becomes available, through a combined global fit.

\subsection{Results in the A2HDM}

\subsubsection{Choice of model parameters}

In the following we assume that the Lagrangian of the scalar sector preserves the $\mathrm{CP}$ symmetry i.e., that the only source of CP violation is still due to the CKM matrix. This makes all the alignment and scalar-potential parameters real. Assuming further that the lightest CP-even scalar $h$ corresponds to the observed neutral boson with $M_{h} \simeq 126 \mathrm{GeV}$, there are ten free parameters in our calculation: three alignment parameters $\varsigma_{f}$, three scalar masses $\left(M_{H}, M_{A}, M_{H^{ \pm}}\right)$, one mixing angle $\tilde{\alpha}$, two scalar-potential couplings $\left(\lambda_{3}, \lambda_{7}\right)$, and the misalignment parameter $C_{R}\left(M_{W}\right)$.

In order to gain insight into the parameter space allowed by $B_{s, d}^{0} \rightarrow \ell^{+} \ell^{-}$decays, it is necessary to take into account information about the $h(126)$ collider data and flavour physics constraints, as well as EW precision observables, which will be crucial for making simplifying assumptions and reducing the number of relevant variables. Explicitly, the following constraints and assumptions on the model parameters are taken into account:

- Firstly, the mixing angle $\tilde{\alpha}$ is constrained at $|\cos \tilde{\alpha}|>0.90$ (68\% CL) through a global fit to the latest LHC and Tevatron data for the $h(126)$ boson [20, 21], which is very close to the SM limit; i.e., the lightest CP-even scalar $h$ behaves like the SM Higgs boson. 
- To assure the validity of perturbative unitarity in the scalar-scalar scattering amplitudes, upper bounds on the quartic Higgs self-couplings are usually imposed by requiring them to be smaller than $8 \pi[8,9]$; i.e., $\left|\lambda_{3,7}\right| \lesssim 8 \pi$.

- With our convention, the lower bound on the heavier CP-even scalar mass is $M_{H} \geq$ $M_{h} \simeq 126 \mathrm{GeV}$. Much lower values of $M_{A}$ are still allowed experimentally. There are, however, no stringent upper limits on these masses. Here we limit them at $M_{H} \in[130,500] \mathrm{GeV}$ and $M_{A} \in[80,500] \mathrm{GeV}$.

- The charged Higgs mass is assumed to lie in the range $M_{H^{ \pm}} \in[80,500] \mathrm{GeV}$, which would require $\left|\varsigma_{u}\right| \leq 2$ to be compatible with the present data on loop-induced processes, such as $Z \rightarrow \bar{b} b, b \rightarrow s \gamma$ and $B_{s, d}^{0}-\bar{B}_{s, d}^{0}$ mixing, as well as the $h(126)$ decays [20, 21, 23-27].

- The alignment parameters $\varsigma_{d}$ and $\varsigma_{\ell}$ are only mildly constrained through phenomenological requirements involving other model parameters. As in our previous works, we restrict them at $\left|\varsigma_{d, \ell}\right| \leq 50[23-27]$.

- At present, there are no useful constraints on the misalignment parameter $C_{R}\left(M_{W}\right)$. For simplicity, it is assumed to be zero.

Numerically, it is found that the ratio $\bar{R}_{s \mu}$ is less sensitive to the scalar-potential couplings $\lambda_{3}$ and $\lambda_{7}$ than to the other model parameters, especially when the alignment parameters are small and/or the neutral scalar masses are large. The mixing angle $\tilde{\alpha}$, when constrained in the range $\cos \tilde{\alpha} \in[0.9,1]$, is also found to have only a marginal impact on $\bar{R}_{s \mu}$. Thus, for simplicity, we shall assign the following values to these parameters:

$$
\lambda_{3}=\lambda_{7}=1, \quad \cos \tilde{\alpha}=0.95 .
$$

As can be seen from eqs. (3.47) and (3.48), the Wilson coefficients $C_{S}$ and $C_{P}$ are always accompanied with the power-suppressed factor $M_{B_{q}}^{2} / M_{W}^{2}$ compared to $C_{10}$. The NP contribution to $C_{10}$ is, however, proportional to $\left|\varsigma_{u}\right|^{2}$ and depends only on the chargedscalar mass. It is, therefore, interesting to discuss the following two special cases with respect to the choice of the alignment parameters: the first one is when $\left|\varsigma_{d, \ell}\right| \lesssim\left|\varsigma_{u}\right| \leq 2$, where the NP contribution is dominated by $C_{10}$ while $C_{S}$ and $C_{P}$ are negligible. The second one is when $\left|\varsigma_{d, \ell}\right| \gg\left|\varsigma_{u}\right|$, which means that $C_{S}$ and $C_{P}$ play a significant role.

\subsubsection{Small $\varsigma_{d, \ell}$}

When the alignment parameters $\varsigma_{d, \ell}$ are of the same size as (or smaller than) $\varsigma_{u}$, the NP contributions from $C_{S}$ and $C_{P}$ are negligible. In this case, we need only to focus on the Wilson coefficient $C_{10}$, which is the sum of the SM contribution $C_{10}^{\mathrm{SM}}$ and the charged-Higgs contribution $C_{10}^{\mathrm{A} 2 \mathrm{HDM}}$ due to $Z$-penguin diagrams shown in figure 5 . The latter involves only two free parameters, $\varsigma_{u}$ and $M_{H^{ \pm}}$, and goes to zero when $\varsigma_{u} \rightarrow 0$ and/or $M_{H^{ \pm}} \rightarrow \infty$.

The dependence of $\bar{R}_{s \mu}$ on the alignment parameter $\varsigma_{u}$ with three typical charged-Higgs masses $(80,200$ and $500 \mathrm{GeV})$ is shown in figure 10 . One can see that, with the contributions from $C_{S}$ and $C_{P}$ ignored, the observable $\bar{R}_{s \mu}$ puts a strong constraint on the parameter $\varsigma_{u}$. 

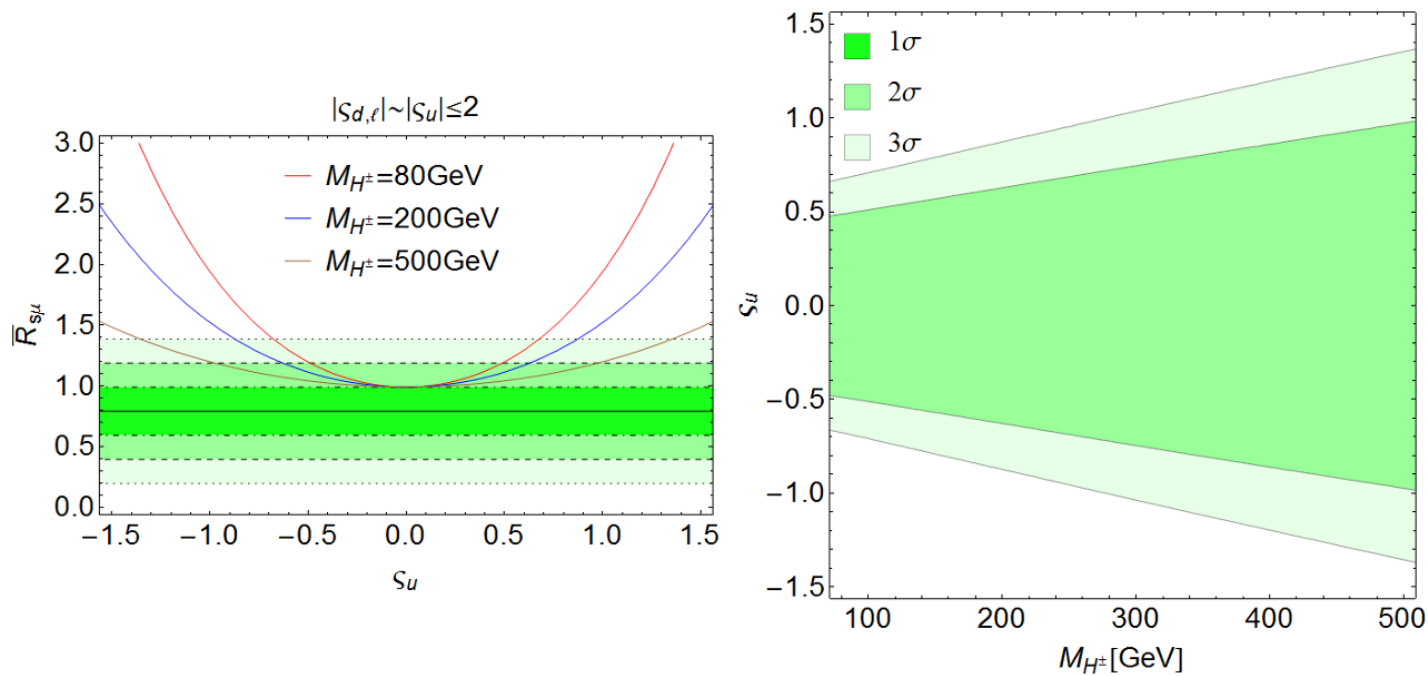

Figure 10. Dependence of $\bar{R}_{s \mu}$ on $\varsigma_{u}$ (left), for $\left|\varsigma_{d, \ell}\right| \lesssim\left|\varsigma_{u}\right| \leq 2$ and $M_{H^{ \pm}}=80,200$ and $500 \mathrm{GeV}$ (upper, middle and lower curves, respectively). The shaded horizontal bands denote the allowed experimental region at $1 \sigma$ (dark green), $2 \sigma$ (green), and $3 \sigma$ (light green), respectively. The right panel shows the resulting upper bounds on $\varsigma_{u}$, as function of $M_{H^{ \pm}}$.

For $M_{H^{ \pm}}=80(500) \mathrm{GeV}$, a 95\% CL upper bound $\left|\varsigma_{u}\right| \leq 0.49$ (0.97) is obtained, with the assumption $\left|\varsigma_{d, \ell}\right| \lesssim\left|\varsigma_{u}\right|$, which is stronger than the constraint from $R_{b}$ [22]. Since $C_{10}^{\mathrm{A} 2 \mathrm{HDM}} \sim\left|\varsigma_{u}\right|^{2}$, this constraint is independent of any assumption about CP and, therefore, applies in the most general case. ${ }^{5}$ For larger charged-Higgs masses, the constraint becomes weaker as the NP effect starts to decouple, reflected by $\lim _{x_{H^{+}} \rightarrow \infty} C_{10}^{\mathrm{A} 2 \mathrm{HDM}}=0$.

\subsubsection{Large $\varsigma_{d, \ell}$}

When $\varsigma_{d}$ and $\varsigma_{\ell}$ are large, the scalar and pseudoscalar operators can induce a significant enhancement of the branching ratio. To see this explicitly, we vary $\varsigma_{d}$ and $\varsigma_{\ell}$ within the range $[-50,50]$, and choose three representative values of $\varsigma_{u}, \varsigma_{u}=0, \pm 1$. We also take three different representative sets of scalar masses:

$$
\begin{aligned}
& \text { Mass } 1: M_{H^{ \pm}}=M_{A}=80 \mathrm{GeV}, \quad M_{H}=130 \mathrm{GeV}, \\
& \text { Mass2 }: M_{H^{ \pm}}=M_{A}=M_{H}=200 \mathrm{GeV}, \\
& \text { Mass3 }: M_{H^{ \pm}}=M_{A}=M_{H}=500 \mathrm{GeV},
\end{aligned}
$$

which cover the lower, intermediate, and upper range, respectively, of the allowed scalar spectrum.

With the above specification, we show in figure 11 the allowed regions in the $\varsigma_{d}-\varsigma_{\ell}$ plane under the constraint from $\bar{R}_{s \mu}$. One can see that, irrespective of the scalar masses, regions with large $\varsigma_{d}$ and $\varsigma_{\ell}$ are already excluded, especially when they have the same sign. The

\footnotetext{
${ }^{5}$ Actually, the explicit correction factor given at the end of eq. (3.52) is valid only in the absence of new sources of $\mathrm{CP}$ violation beyond the SM. Taking the correct general relation into account, the upperbounded parameter is $\left|\varsigma_{u}\right|\left\{\left[1+y_{s} \cos \left(2 \phi_{P}-\phi_{s}^{\mathrm{NP}}\right)\right] /\left(1+y_{s}\right)\right\}^{1 / 4} \approx\left|\varsigma_{u}\right|$, where the phase $\phi_{s}^{\mathrm{NP}}$ denotes the CP-violating NP contribution to $B_{s}^{0}-\bar{B}_{s}^{0}$ mixing.
} 

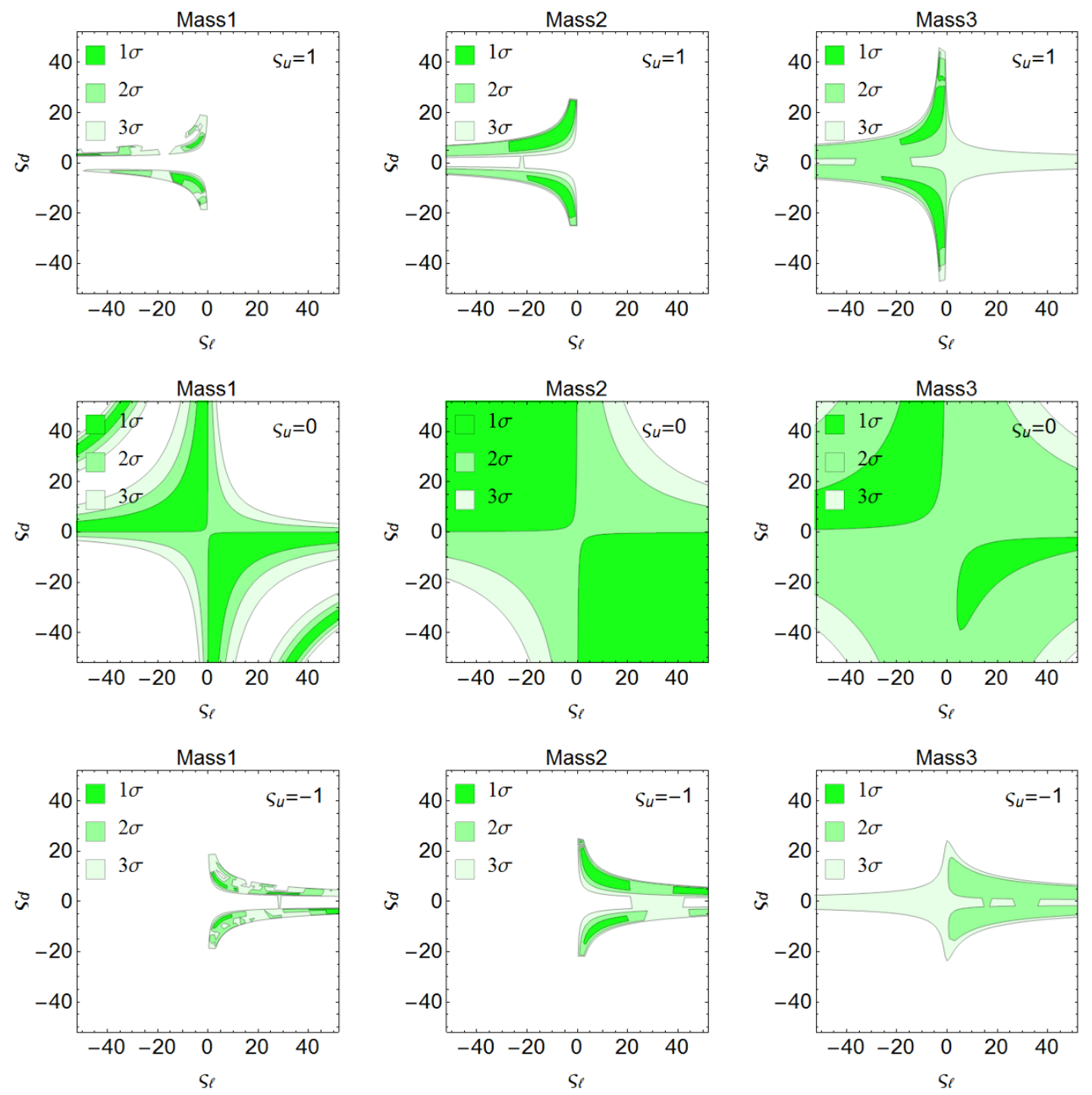

Figure 11. Allowed regions (at $1 \sigma, 2 \sigma$ and $3 \sigma$ ) in the $\varsigma_{d}{ }^{-} \varsigma_{\ell}$ plane under the constraint from $\bar{R}_{s \mu}$, with three different assignments of the scalar masses and $\varsigma_{u}=0, \pm 1$.

impact of $\varsigma_{u}$, even when varied within the small range $[-1,1]$, is found to be significant: a nonzero $\varsigma_{u}$ will exclude most of the regions allowed in the case with $\varsigma_{u}=0$, and changing the sign of $\varsigma_{u}$ will also flip that of $\varsigma_{\ell}$. This is mainly due to the factors $\varsigma_{d}^{2} \varsigma_{u}^{*}$ appearing in the functions $g_{2}^{(a)}$ and $g_{3}^{(a)}$ defined, respectively, by eqs. (A.29) and (A.30). It is also observed that the allowed regions expand with increasing scalar masses, as expected, since larger scalar masses make the NP contributions gradually decouple from the SM.

\section{$4.4 \quad \mathcal{Z}_{2}$ symmetric models}

The five types of $\mathcal{Z}_{2}$-symmetric models listed in Table 1 are particular cases of the CPconserving $\mathrm{A} 2 \mathrm{HDM}$, with the three alignment factors $\varsigma_{f}$ reduced to a single parameter $\tan \beta=v_{2} / v_{1} \geq 0$. In the particular scalar basis where the discrete $\mathcal{Z}_{2}$ symmetry is 

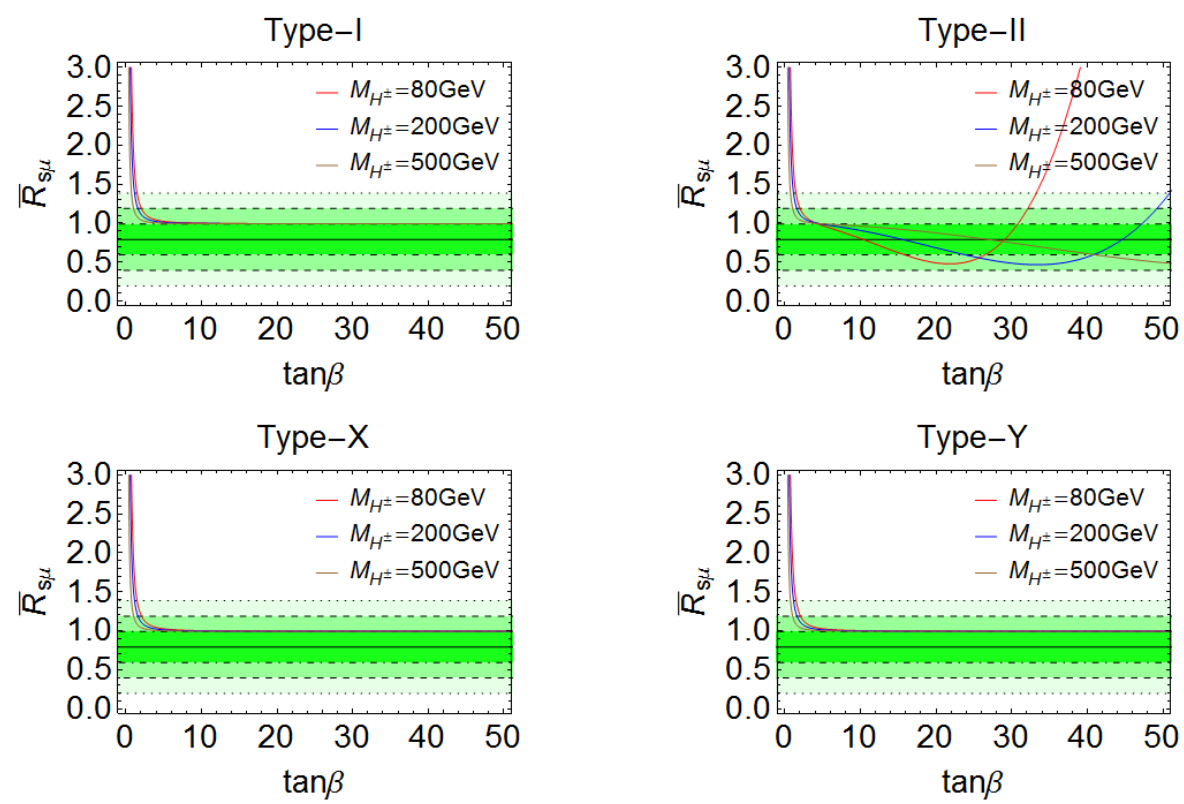

Figure 12. Dependence of $\bar{R}_{s \mu}$ on $\tan \beta$ for the 2HDMs of types I, II, X and Y. The upper, middle and lower curves correspond to $M_{H^{ \pm}}=80,200$ and $500 \mathrm{GeV}$, respectively. The horizontal bands denote the allowed experimental region at $1 \sigma$ (dark green), $2 \sigma$ (green), and $3 \sigma$ (light green), respectively.

implemented, the scalar-potential couplings $\mu_{i}^{\prime}$ and $\lambda_{i}^{\prime}$ must be real, and $\mu_{3}^{\prime}=\lambda_{6}^{\prime}=\lambda_{7}^{\prime}=0$; however, the rotation into the Higgs basis generates non-zero values of $\mu_{3}=-\frac{1}{2} \lambda_{6} v^{2}$ and $\lambda_{7}$. Furthermore, the alignment condition is protected by the $\mathcal{Z}_{2}$ symmetry at any energy scale, which means that the misalignment parameter $C_{R}\left(M_{W}\right)$ does not contribute and the Higgs-penguin diagrams are free of divergences. Thus, for $\mathcal{Z}_{2}$-symmetric models, the ratio $\bar{R}_{s \mu}$ only involves seven free parameters: $M_{H^{ \pm}}, M_{H}, M_{A}, \lambda_{3}, \lambda_{7}, \cos \tilde{\alpha}$, and $\tan \beta$.

A much more constrained case is the inert $2 \mathrm{HDM}$, where the $\mathcal{Z}_{2}$ symmetry is imposed in the Higgs basis: all SM fields and $\Phi_{1}$ are even while $\Phi_{2} \rightarrow-\Phi_{2}$ under the $\mathcal{Z}_{2}$ transformation. This implies that there is no mixing between the CP-even neutral states $h$ and $H$, and the scalars $H, A$ and $H^{ \pm}$decouple from the fermions: $\cos \tilde{\alpha}=1, \lambda_{6}=\lambda_{7}=0, \varsigma_{f}=0$. Moreover, the couplings of $h$ to fermions and vector bosons are identical to the SM ones. Therefore, in the inert model $\bar{R}_{s \mu}^{\text {inert }}=1$.

For the other four types of $\mathcal{Z}_{2}$-symmetric models, we continue to use the assignments $\cos \tilde{\alpha}=0.95$ and $\lambda_{3}=\lambda_{7}=1$. One can easily check that the effects of $M_{H}$ and $M_{A}$ on $\bar{R}_{s \mu}$ are tiny, unless $\tan \beta$ is extremely small which is excluded by the flavour constraint $\left|\varsigma_{u}\right| \leq 2$. For simplicity, we fix them to be $M_{H}=M_{A}=500 \mathrm{GeV}$ in the following analysis.

Figure 12 shows the dependence of $\bar{R}_{s \mu}$ on the parameter $\tan \beta$, for three representative values of the charged-Higgs mass: $M_{H^{ \pm}}=80,200$ and $500 \mathrm{GeV}$. The four different panels correspond to the $\mathcal{Z}_{2}$-symmetric models of types I, II, X and Y, respectively. A lower bound $\tan \beta>1.6$ is obtained at $95 \%$ CL under the constraint from the current experimental data on $\bar{R}_{s \mu}$. This implies $\varsigma_{u}=\cot \beta<0.63$, which is stronger than the bounds obtained previously from other sources [22-27]. 
One can see that the predicted $\bar{R}_{s \mu}$ in the type-I, type-X and type-Y models are almost indistinguishable from each other and, in the large $\tan \beta$ region, approach the SM prediction, irrespective of the choices of scalar masses. For the type-II model, on the other hand, an enhancement of $\bar{R}_{s \mu}$ is still possible in the large $\tan \beta$ region. This can be understood since the Wilson coefficients in the type-II model contain the factor $\tan ^{2} \beta$ arising from the product of alignment parameters $\varsigma_{f}$, while in the other three models they contain at most one power of $\tan \beta$. So only the type-II model can receive a large $\tan \beta$ enhancement, which has been studied intensively in the literature [28-30]. It is also interesting to note that in the type-II $2 \mathrm{HDM}$ with large $\tan \beta$ the $B_{s, d}^{0} \rightarrow \ell^{+} \ell^{-}$branching ratios depend only on the charged-Higgs mass and $\tan \beta[28]$.

\section{Conclusions}

In this paper, we have performed a detailed analysis of the rare decays $B_{s, d}^{0} \rightarrow \ell^{+} \ell^{-}$within the general framework of the A2HDM. Firstly, we presented a complete one-loop calculation of the short-distance Wilson coefficients $C_{10}, C_{S}$ and $C_{P}$, which arise from various box and penguin diagrams, and made a detailed technical summary of our results and a comparison with previous calculations performed in particular limits or approximations. In order to make sure our results are gauge independent, the calculations were carried out in both the Feynman and the unitary gauges.

With the current data on $\overline{\mathcal{B}}\left(B_{s}^{0} \rightarrow \mu^{+} \mu^{-}\right)$taken into account, we have also investigated the impact of various model parameters on the branching ratios and studied the phenomenological constraints imposed by present data. The resulting information about the model parameters will be crucial for the model building and is complementary to the collider searches for new scalar resonances in the near future.

When $\left|\varsigma_{d, \ell}\right| \lesssim\left|\varsigma_{u}\right|$, the contributions to $\overline{\mathcal{B}}\left(B_{s}^{0} \rightarrow \mu^{+} \mu^{-}\right)$from the scalar and pseudoscalar operators are negligible compared to the leading Wilson coefficient $C_{10}$. Since $C_{10}^{\mathrm{A} 2 \mathrm{HDM}} \sim\left|\varsigma_{u}\right|^{2}$, the measured $\overline{\mathcal{B}}\left(B_{s}^{0} \rightarrow \mu^{+} \mu^{-}\right)$branching ratio implies then an upper bound on the up-family alignment parameter, which only depends on the charged Higgs mass. At $95 \%$ CL, we obtain:

$$
\left|\varsigma_{u}\right| \leq 0.49(0.97), \quad \text { for } \quad M_{H^{ \pm}}=80(500) \mathrm{GeV} \quad \text { and } \quad\left|\varsigma_{d, \ell}\right| \lesssim\left|\varsigma_{u}\right| .
$$

This bound is stronger than the constraints obtained previously from other sources [22-27].

The role of the scalar and pseudoscalar operators becomes much more important for large values of $\left|\varsigma_{d, \ell}\right|$. This region of parameter space was previously explored within the context of the type-II $2 \mathrm{HDM}$, where these contributions are enhanced by a factor $\tan ^{2} \beta$. Our analysis agrees with previous results in the type-II case and shows, moreover, that this $\tan ^{2} \beta$ enhancement is absent in the $\mathcal{Z}_{2}$-symmetric models of types I, X and Y, which approach the SM prediction for large values of $\tan \beta$. From the current experimental data on $\bar{R}_{s \mu}$, we derive the $95 \%$ CL bound:

$$
\tan \beta>1.6, \quad \text { for } 2 \text { HDMs of types I, II, } \mathrm{X} \text { and } \mathrm{Y} \text {. }
$$

This implies $\varsigma_{u}=\cot \beta<0.63$, which is also stronger than the bounds obtained previously from other sources [22-27]. 
The enhancement of the scalar and pseudoscalar contributions at large values of $\left|\varsigma_{d, \ell}\right|$ is present in the most general A2HDM scenario and could give rise to interesting phenomenological signals. To exemplify this possibility, we have analyzed the ratio $\bar{R}_{s \mu}$ in the simpler CP-conserving case, showing the important impact of the A2HDM corrections whenever enhanced Yukawa couplings to leptons and down-type quarks are present. The resulting constraints on the alignment parameters are given in figure 11.

It would be interesting to analyze the possible impact of the new CP-violating phases present within the A2HDM framework, at large values of $\left|\varsigma_{d, \ell}\right|$. They could generate sizeable phases $\phi_{P}$ and $\phi_{S}$ in eqs. (3.47) and (3.48), which could manifest themselves in the timedependence of the $B_{s}^{0} \rightarrow \mu^{+} \mu^{-}$decay amplitude [71]. To quantify the possible size of this effect requires a more careful assessment of the allowed parameter space of the A2HDM, which we plan to further investigate in future works.

\section{Acknowledgments}

We are grateful to Alejandro Celis and Victor Ilisie for useful discussions on the 2HDM parameters. This work was supported in part by the National Natural Science Foundation of China (NSFC) under contract No. 11005032, the Spanish Government and ERDF funds from the EU Commission [Grants FPA2011-23778 and CSD2007-00042 (Consolider Project CPAN)] and by Generalitat Valenciana under Grant No. PROMETEOII/2013/007. X. Q. Li was also supported by the Specialized Research Fund for the Doctoral Program of Higher Education of China (Grant No. 20104104120001) and by the Scientific Research Foundation for the Returned Overseas Chinese Scholars, State Education Ministry.

\section{A Scalar-penguin results within the A2HDM}

The coefficients $\hat{C}^{\varphi_{i}^{0}}$, defined in eq. (3.33), are given by

$$
\hat{C}^{\varphi_{i}^{0}}=\frac{m_{t}^{2}}{M_{\varphi_{i}^{0}}^{2}}\left\{\sum_{k=1}^{18} C^{k, \varphi_{i}^{0}}+\frac{1}{2}\left(\varsigma_{u}-\varsigma_{d}\right)\left(1+\varsigma_{u}^{*} \varsigma_{d}\right)\left(\mathcal{R}_{i 2}+i \mathcal{R}_{i 3}\right) \mathcal{C}\right\},
$$

where the last term is the tree-level contribution from the local operator in eq. (2.15). We detail next the contributions $C^{k, \varphi_{i}^{0}}$ from the separate diagrams $(k=1, \cdots, 18)$ shown in figure 9.

The gauge-independent coefficients are:

$$
\begin{aligned}
C^{1, \varphi_{i}^{0}}= & \frac{y_{u}^{\varphi_{i}^{0}}}{4}\left\{\varsigma_{d} \varsigma_{u}^{*} \frac{x_{t}}{x_{H^{+}}-x_{t}}\left[1-\frac{x_{H^{+}}}{x_{H^{+}}-x_{t}}\left(\ln x_{H^{+}}-\ln x_{t}\right)\right]\right. \\
& \left.+\left|\varsigma_{u}\right|^{2} \frac{x_{t}}{2\left(x_{H^{+}}-x_{t}\right)^{2}}\left[\frac{3 x_{t}-x_{H^{+}}}{2}+\frac{x_{H^{+}}\left(x_{H^{+}}-2 x_{t}\right)}{x_{H^{+}}-x_{t}}\left(\ln x_{H^{+}}-\ln x_{t}\right)\right]\right\} \\
& +\frac{y_{u}^{\varphi_{i}^{0}}}{4}\left\{\varsigma_{d} \varsigma_{u}^{*}\left[\Lambda+\frac{x_{t}}{x_{H^{+}}-x_{t}}-\frac{x_{H^{+}}^{2}}{\left(x_{H^{+}}-x_{t}\right)^{2}} \ln x_{H^{+}}+\frac{x_{t}\left(2 x_{H^{+}}-x_{t}\right)}{\left(x_{H^{+}}-x_{t}\right)^{2}} \ln x_{t}\right]\right. \\
& \left.+\left|\varsigma_{u}\right|^{2} \frac{x_{t}}{2\left(x_{H^{+}}-x_{t}\right)^{2}}\left[\frac{3 x_{H^{+}}-x_{t}}{2}-\frac{x_{H^{+}}^{2}}{x_{H^{+}}-x_{t}}\left(\ln x_{H^{+}}-\ln x_{t}\right)\right]\right\},
\end{aligned}
$$




$$
\begin{aligned}
C^{2, \varphi_{i}^{0}}= & \frac{s_{W}^{2} \lambda_{H^{+} H^{-}}^{\varphi^{0}}}{4 \pi \alpha\left(x_{H^{+}}-x_{t}\right)}\left\{\varsigma_{d} \varsigma_{u}^{*}\left[\frac{x_{t}}{x_{H^{+}}-x_{t}}\left(\ln x_{H^{+}}-\ln x_{t}\right)-1\right]\right. \\
& \left.+\left|\varsigma_{u}\right|^{2}\left[\frac{x_{t}^{2}}{2\left(x_{H^{+}}-x_{t}\right)^{2}}\left(\ln x_{H^{+}}-\ln x_{t}\right)+\frac{x_{H^{+}}-3 x_{t}}{4\left(x_{H^{+}}-x_{t}\right)}\right]\right\}, \\
C^{3, \varphi_{i}^{0}}= & \frac{y_{d}^{\varphi_{i}^{0}}}{4} \varsigma_{d} \varsigma_{u}^{*}\left[-\Lambda+\frac{x_{H^{+}}}{x_{H^{+}}-x_{t}} \ln x_{H^{+}}-\frac{x_{t}}{x_{H^{+}}-x_{t}} \ln x_{t}\right], \\
C^{4, \varphi_{i}^{0}}= & C^{7, \varphi_{i}^{0}}=C^{8, \varphi_{i}^{0}}=0 .
\end{aligned}
$$

In the unitary gauge, we find:

$$
\begin{aligned}
C_{\text {Unitary }}^{5, \varphi_{i}^{0}}= & \frac{1}{4}\left\{y_{u}^{\varphi_{i}^{0} *}\left[\Lambda-\frac{5 x_{t}^{2}-13 x_{t}+2}{4\left(x_{t}-1\right)^{2}}-\frac{2 x_{t}^{3}-6 x_{t}^{2}+9 x_{t}-2}{2\left(x_{t}-1\right)^{3}} \ln x_{t}\right]\right. \\
& \left.+y_{u}^{\varphi_{i}^{0}}\left[\frac{\Lambda}{2}-\frac{2 x_{t}^{2}-x_{t}-7}{4\left(x_{t}-1\right)^{2}}-\frac{x_{t}^{3}-3 x_{t}^{2}+3 x_{t}+2}{2\left(x_{t}-1\right)^{2}} \ln x_{t}\right]\right\}, \\
C_{\text {Unitary }}^{6, \varphi_{i}^{0}}= & \frac{\lambda_{W^{+} W^{-}}^{\varphi_{i}^{0}}}{8}\left[-3 \Lambda+\frac{x_{t}^{2}-2 x_{t}-11}{2\left(x_{t}-1\right)^{2}}+\frac{3 x_{t}\left(x_{t}^{2}-3 x_{t}+4\right)}{\left(x_{t}-1\right)^{3}} \ln x_{t}\right], \\
C_{\text {Unitary }}^{9, \varphi_{i}^{0}}= & \left.\frac{\lambda_{H^{+} W^{-}}^{\varphi^{0}}}{8} \varsigma_{u}^{*}\left[\frac{1}{2}-\Lambda+\frac{x_{H^{+}}\left(x_{H^{+}}+2\right) \ln x_{H^{+}}}{\left(x_{H^{+}}-1\right)\left(x_{H^{+}}-x_{t}\right)}-\frac{x_{t}\left(x_{t}+2\right) \ln x_{t}}{\left(x_{t}-1\right)\left(x_{H^{+}}-x_{t}\right)}\right], \quad \text { (A. }\right) \\
C_{\mathrm{Unitary}}^{10, \varphi_{i}^{0}}= & \frac{\lambda_{H^{+} W^{-}}^{\varphi^{0} *}}{4}\left\{\varsigma_{d}\left[-\Lambda+\frac{x_{H^{+}} \ln x_{H^{+}}}{x_{H^{+}}-x_{t}}-\frac{x_{t} \ln x_{t}}{x_{H^{+}}-x_{t}}\right]-\frac{\varsigma_{u}}{2}\left[\frac{x_{t}\left(x_{H^{+}} x_{t}-4 x_{H^{+}}+3 x_{t}\right)}{\left(x_{t}-1\right)\left(x_{H^{+}}-x_{t}\right)^{2}} \ln x_{t}\right.\right. \\
& \left.\left.+\frac{x_{H^{+}}}{x_{H^{+}}-x_{t}}-\frac{x_{H^{+}}\left(x_{H^{+}} x_{t}-3 x_{H^{+}}+2 x_{t}\right)}{\left(x_{H^{+}}-1\right)\left(x_{H^{+}}-x_{t}\right)^{2}} \ln x_{H^{+}}\right]\right\} .
\end{aligned}
$$

In the Feynman gauge the results are:

$$
\begin{aligned}
C_{\mathrm{Feynman}}^{5, \varphi_{i}^{0}}= & \frac{1}{8\left(x_{t}-1\right)^{2}}\left\{y_{u}^{\varphi_{i}^{0 *}}\left[3 x_{t}-1+\frac{2\left(1-2 x_{t}\right) \ln x_{t}}{x_{t}-1}\right]+y_{u}^{\varphi_{i}^{0}}\left[3-x_{t}-\frac{2 \ln x_{t}}{x_{t}-1}\right]\right\}, \text { (A.10) } \\
C_{\mathrm{Feynman}}^{6, \varphi_{i}^{0}}= & \frac{\lambda_{W^{+} W^{-}}^{\varphi_{i}^{0}}}{4\left(x_{t}-1\right)^{2}}\left[\frac{2 x_{t}}{x_{t}-1} \ln x_{t}-x_{t}-1\right], \\
C_{\mathrm{Feynman}}^{9, \varphi_{i}^{0}}= & \frac{\varsigma_{u}^{*} \lambda_{H^{+} W^{-}}^{\varphi^{0}}}{8\left(x_{H^{+}}-x_{t}\right)}\left[\frac{x_{H^{+}}-x_{t}}{\left(x_{H^{+}}-1\right)\left(x_{t}-1\right)}+\frac{x_{H^{+}}\left(3 x_{H^{+}}-2\right) \ln x_{H^{+}}}{\left(x_{H^{+}}-1\right)^{2}}-\frac{x_{t}\left(3 x_{t}-2\right) \ln x_{t}}{\left(x_{t}-1\right)^{2}}\right], \\
C_{\mathrm{Feynman}}^{10, \varphi_{i}^{0}}= & \frac{\lambda_{H^{+} W^{-}}^{\varphi^{0}}}{4\left(x_{H^{+}}-x_{t}\right)}\left\{\varsigma_{d}\left[\frac{x_{t} \ln x_{t}}{x_{t}-1}-\frac{x_{H^{+}} \ln x_{H^{+}}}{x_{H^{+}}-1}\right]+\frac{\varsigma_{u}}{2}\left[\frac{x_{H^{+}}}{x_{H^{+}}-1}+\frac{x_{t}\left(4 x_{H^{+}}-3 x_{t}\right) \ln x_{t}}{\left(x_{t}-1\right)\left(x_{H^{+}}-x_{t}\right)}\right.\right. \\
& \left.\left.-\frac{x_{H^{+}}\left(4 x_{H^{+}}^{2}-3 x_{H^{+}} x_{t}-3 x_{H^{+}}+2 x_{t}\right)}{\left(x_{H^{+}}-1\right)^{2}\left(x_{H^{+}}-x_{t}\right)} \ln x_{H^{+}}\right]\right\},
\end{aligned}
$$




$$
\begin{aligned}
& C_{\text {Feynman }}^{11, \varphi_{i}^{0}}=\frac{1}{4}\left\{y_{u}^{\varphi_{i}^{0} *}\left[\Lambda-\frac{x_{t}\left(5 x_{t}-7\right)}{4\left(x_{t}-1\right)^{2}}-\frac{x_{t}\left(2 x_{t}^{2}-6 x_{t}+5\right)}{2\left(x_{t}-1\right)^{3}} \ln x_{t}\right]\right. \\
& \left.-\frac{y_{u}^{\varphi_{i}^{0}}}{2}\left[\frac{x_{t}\left(x_{t}-3\right)}{2\left(x_{t}-1\right)^{2}}+\frac{x_{t}}{\left(x_{t}-1\right)^{3}} \ln x_{t}\right]\right\} \\
& C_{\text {Feynman }}^{12, \varphi_{i}^{0}}=\frac{s_{W}^{2} \lambda_{G^{+} G^{-}}^{\varphi_{0}^{0}}}{16 \pi \alpha\left(x_{t}-1\right)^{2}}\left[x_{t}-3-\frac{2 x_{t}\left(x_{t}-2\right)}{x_{t}-1} \ln x_{t}\right] \\
& C_{\text {Feynman }}^{13, \varphi_{i}^{0}}=\frac{y_{d}^{\varphi_{i}^{0}}}{4}\left[-\Lambda+\frac{x_{t}}{x_{t}-1} \ln x_{t}\right], \\
& C_{\text {Feynman }}^{14, \varphi_{i}^{0}}=0, \\
& C_{\mathrm{Feynman}}^{15, \varphi_{i}^{0}}=\frac{s_{W}^{2} \varsigma_{u}^{*} \lambda_{H^{+} G^{-}}^{\varphi^{0}}}{8 \pi \alpha\left(x_{H^{+}}-x_{t}\right)}\left[\frac{x_{H^{+}}-x_{t}}{\left(x_{H^{+}}-1\right)\left(x_{t}-1\right)}+\frac{x_{t}\left(x_{t}-2\right)}{\left(x_{t}-1\right)^{2}} \ln x_{t}-\frac{x_{H^{+}}\left(x_{H^{+}}-2\right)}{\left(x_{H^{+}}-1\right)^{2}} \ln x_{H^{+}}\right], \\
& C_{\text {Feynman }}^{16, \varphi_{i}^{0}}=\frac{s_{W^{2}}^{2} \lambda_{H^{+} G^{-}}^{\varphi^{*}}}{8 \pi \alpha\left(x_{H^{+}}-x_{t}\right)}\left\{2 \varsigma_{d}\left[\frac{x_{t}}{x_{t}-1} \ln x_{t}-\frac{x_{H^{+}}}{x_{H^{+}}-1} \ln x_{H^{+}}\right]+\varsigma_{u}\left[\frac{x_{t}^{2} \ln x_{t}}{\left(x_{t}-1\right)\left(x_{H^{+}}-x_{t}\right)}\right.\right. \\
& \left.\left.+\frac{x_{H^{+}}}{x_{H^{+}}-1}-\frac{x_{H^{+}}\left(x_{H^{+}} x_{t}+x_{H^{+}}-2 x_{t}\right)}{\left(x_{H^{+}}-1\right)^{2}\left(x_{H^{+}}-x_{t}\right)} \ln x_{H^{+}}\right]\right\} \\
& C_{\text {Feynman }}^{17, \varphi_{i}^{0}}=\frac{\lambda_{G^{+} W^{-}}^{\varphi^{0}}}{8\left(x_{t}-1\right)^{2}}\left[\frac{5-7 x_{t}}{2}+\frac{x_{t}\left(3 x_{t}-2\right)}{x_{t}-1} \ln x_{t}\right] \text {, } \\
& C_{\text {Feynman }}^{18, \varphi_{i}^{0}}=\frac{\lambda_{G^{+} W^{-}}^{\varphi^{0}}}{8\left(x_{t}-1\right)^{2}}\left[\frac{9 x_{t}-11}{2}-\frac{x_{t}\left(5 x_{t}-6\right)}{x_{t}-1} \ln x_{t}\right] \text {. }
\end{aligned}
$$

Here $\Lambda=-\frac{2 \mu^{D-4}}{D-4}-\gamma_{E}+\ln (4 \pi)-\ln \left(\frac{M_{W}^{2}}{\mu^{2}}\right)+1$, and the (rescaled) cubic coupling constants are defined, respectively, as

$$
\begin{aligned}
\lambda_{W^{+} W^{-}}^{\varphi^{0}} & =\lambda_{G^{+} W^{-}}^{\varphi^{0}}=\mathcal{R}_{i 1}, \\
\lambda_{H_{i}^{+} W^{-}}^{\varphi^{0}} & =\mathcal{R}_{i 2}-i \mathcal{R}_{i 3}, \\
\lambda_{H^{+} H^{-}}^{\varphi^{0}} & =\lambda_{3} \mathcal{R}_{i 1}+\lambda_{7}^{R} \mathcal{R}_{i 2}-\lambda_{7}^{I} \mathcal{R}_{i 3}, \\
\lambda_{G^{+} G^{-}}^{\varphi^{0}} & =2 \lambda_{1} \mathcal{R}_{i 1}+\lambda_{6}^{R} \mathcal{R}_{i 2}-\lambda_{6}^{I} \mathcal{R}_{i 3}=\frac{M_{\varphi_{i}^{0}}^{2}}{v^{2}} \mathcal{R}_{i 1}, \\
\lambda_{H^{+} G^{-}}^{\varphi^{0}} & =\lambda_{6} \mathcal{R}_{i 1}+\frac{1}{2}\left(\lambda_{4}+2 \lambda_{5}\right) \mathcal{R}_{i 2}-\frac{i}{2}\left(\lambda_{4}-2 \lambda_{5}\right) \mathcal{R}_{i 3}=\frac{M_{\varphi_{i}^{0}}^{2}-M_{H^{+}}^{2}}{v^{2}}\left(\mathcal{R}_{i 2}-i \mathcal{R}_{i 3}\right) .
\end{aligned}
$$

The functions $g_{0}\left(x_{t}, x_{H^{+}}, \varsigma_{u}, \varsigma_{d}\right)$ and $g_{j}^{(a)}\left(x_{t}, x_{H^{+}}, \varsigma_{u}, \varsigma_{d}\right)$ introduced in eq. (3.34) are gauge independent. For $g_{0}\left(x_{t}, x_{H^{+}}, \varsigma_{u}, \varsigma_{d}\right)$ we find

$$
g_{0}\left(x_{t}, x_{H^{+}}, \varsigma_{u}, \varsigma_{d}\right)=\frac{\pi \alpha C^{2, \varphi_{i}^{0}}}{s_{W}^{2} \lambda_{H^{+} H^{-}}^{\varphi^{0}}},
$$


while the functions $g_{j}^{(a)}\left(x_{t}, x_{H^{+}}, \varsigma_{u}, \varsigma_{d}\right)$ are given, respectively, as:

$$
\begin{aligned}
g_{1}^{(a)}\left(x_{t}, x_{H^{+}}, \varsigma_{u}, \varsigma_{d}\right)= & -\frac{3}{4}+\varsigma_{d} \varsigma_{u}^{*} \frac{x_{t}}{x_{H^{+}}-x_{t}}\left[1-\frac{x_{H^{+}}}{x_{H^{+}}-x_{t}}\left(\ln x_{H^{+}}-\ln x_{t}\right)\right] \\
& +\left|\varsigma_{u}\right|^{2} \frac{x_{t}}{2\left(x_{H^{+}}-x_{t}\right)^{2}}\left[\frac{x_{H^{+}}+x_{t}}{2}-\frac{x_{H^{+}} x_{t}}{x_{H^{+}}-x_{t}}\left(\ln x_{H^{+}}-\ln x_{t}\right)\right], \\
g_{2}^{(a)}\left(x_{t}, x_{H^{+}}, \varsigma_{u}, \varsigma_{d}\right)= & \varsigma_{d}^{2} \varsigma_{u}^{*} f_{1}\left(x_{t}, x_{H^{+}}\right)+\varsigma_{d}\left(\varsigma_{u}^{*}\right)^{2} f_{2}\left(x_{t}, x_{H^{+}}\right) \\
& +\varsigma_{d}\left|\varsigma_{u}\right|^{2} f_{3}\left(x_{t}, x_{H^{+}}\right)+\varsigma_{u}\left|\varsigma_{u}\right|^{2} f_{4}\left(x_{t}, x_{H^{+}}\right)-\varsigma_{u}^{*}\left|\varsigma_{u}\right|^{2} f_{5}\left(x_{t}, x_{H^{+}}\right) \\
& +\varsigma_{u} f_{6}\left(x_{t}, x_{H^{+}}\right)-\varsigma_{u}^{*} f_{7}\left(x_{t}, x_{H^{+}}\right)+\varsigma_{d} f_{1}\left(x_{t}, x_{H^{+}}\right) \\
g_{3}^{(a)}\left(x_{t}, x_{H^{+}}, \varsigma_{u}, \varsigma_{d}\right)= & \varsigma_{d}^{2} \varsigma_{u}^{*} f_{1}\left(x_{t}, x_{H^{+}}\right)-\varsigma_{d}\left(\varsigma_{u}^{*}\right)^{2} f_{2}\left(x_{t}, x_{H^{+}}\right) \\
& +\varsigma_{d}\left|\varsigma_{u}\right|^{2} f_{3}\left(x_{t}, x_{H^{+}}\right)+\varsigma_{u}\left|\varsigma_{u}\right|^{2} f_{4}\left(x_{t}, x_{H^{+}}\right)+\varsigma_{u}^{*}\left|\varsigma_{u}\right|^{2} f_{5}\left(x_{t}, x_{H^{+}}\right) \\
& +\varsigma_{u} f_{6}\left(x_{t}, x_{H^{+}}\right)+\varsigma_{u}^{*} f_{7}\left(x_{t}, x_{H^{+}}\right)+\varsigma_{d} f_{1}\left(x_{t}, x_{H^{+}}\right) .
\end{aligned}
$$

The functions $g_{j}^{(b)}\left(x_{t}, x_{H^{+}}, \varsigma_{u}, \varsigma_{d}\right)$ are zero in the unitary gauge, because they are all related to Goldstone boson vertices. In the Feynman gauge, they are given, respectively, as

$$
\begin{aligned}
& g_{1, \text { Feynman }}^{(b)}\left(x_{t}, x_{H^{+}}, \varsigma_{u}, \varsigma_{d}\right)=\frac{1}{8\left(x_{t}-1\right)^{2}}\left[\frac{x_{t}-3}{2}-\frac{x_{t}\left(x_{t}-2\right)}{x_{t}-1} \ln x_{t}\right], \\
& g_{2, \text { Feynman }}^{(b)}\left(x_{t}, x_{H^{+}}, \varsigma_{u}, \varsigma_{d}\right)=\varsigma_{d} f_{8}\left(x_{t}, x_{H^{+}}\right)+\varsigma_{u} f_{9}\left(x_{t}, x_{H^{+}}\right)+\varsigma_{u}^{*} f_{10}\left(x_{t}, x_{H^{+}}\right), \\
& g_{3, \text { Feynman }}^{(b)}\left(x_{t}, x_{H^{+}}, \varsigma_{u}, \varsigma_{d}\right)=\varsigma_{d} f_{8}\left(x_{t}, x_{H^{+}}\right)+\varsigma_{u} f_{9}\left(x_{t}, x_{H^{+}}\right)-\varsigma_{u}^{*} f_{10}\left(x_{t}, x_{H^{+}}\right) .
\end{aligned}
$$

Here the functions $f_{j}\left(x_{t}, x_{H^{+}}\right)$are defined, respectively, as

$$
\begin{aligned}
f_{1}\left(x_{t}, x_{H^{+}}\right)= & \frac{1}{2\left(x_{H^{+}}-x_{t}\right)}\left[-x_{H^{+}}+x_{t}+x_{H^{+}} \ln x_{H^{+}}-x_{t} \ln x_{t}\right] \\
f_{2}\left(x_{t}, x_{H^{+}}\right)= & \frac{1}{2\left(x_{H^{+}}-x_{t}\right)}\left[x_{t}-\frac{x_{H^{+}} x_{t}}{x_{H^{+}}-x_{t}}\left(\ln x_{H^{+}}-\ln x_{t}\right)\right] \\
f_{3}\left(x_{t}, x_{H^{+}}\right)= & \frac{1}{2\left(x_{H^{+}}-x_{t}\right)}\left[x_{H^{+}}-\frac{x_{H^{+}}^{2} \ln x_{H^{+}}}{x_{H^{+}}-x_{t}}+\frac{x_{t}\left(2 x_{H^{+}}-x_{t}\right) \ln x_{t}}{x_{H^{+}}-x_{t}}\right] \\
f_{4}\left(x_{t}, x_{H^{+}}\right)= & \frac{1}{4\left(x_{H^{+}}-x_{t}\right)^{2}}\left[\frac{x_{t}\left(3 x_{H^{+}}-x_{t}\right)}{2}-\frac{x_{H^{+}}^{2} x_{t}}{x_{H^{+}}-x_{t}}\left(\ln x_{H^{+}}-\ln x_{t}\right)\right] \\
f_{5}\left(x_{t}, x_{H^{+}}\right)= & \frac{1}{4\left(x_{H^{+}}-x_{t}\right)^{2}}\left[\frac{x_{t}\left(x_{H^{+}}-3 x_{t}\right)}{2}-\frac{x_{H^{+}} x_{t}\left(x_{H^{+}}-2 x_{t}\right)}{x_{H^{+}}-x_{t}}\left(\ln x_{H^{+}}-\ln x_{t}\right)\right] \\
f_{6}\left(x_{t}, x_{H^{+}}\right)= & \frac{1}{2\left(x_{H^{+}}-x_{t}\right)}\left[\frac{x_{t}\left(x_{t}^{2}-3 x_{H^{+}} x_{t}+9 x_{H^{+}}-5 x_{t}-2\right)}{4\left(x_{t}-1\right)^{2}}\right. \\
& +\frac{x_{H^{+}}\left(x_{H^{+}} x_{t}-3 x_{H^{+}}+2 x_{t}\right)}{2\left(x_{H^{+}}-1\right)\left(x_{H^{+}}-x_{t}\right)} \ln x_{H^{+}} \\
+ & \left.\frac{x_{H^{+}}^{2}\left(-2 x_{t}^{3}+6 x_{t}^{2}-9 x_{t}+2\right)+3 x_{H^{+}} x_{t}^{2}\left(x_{t}^{2}-2 x_{t}+3\right)-x_{t}^{2}\left(2 x_{t}^{3}-3 x_{t}^{2}+3 x_{t}+1\right)}{2\left(x_{t}-1\right)^{3}\left(x_{H^{+}}-x_{t}\right)} \ln x_{t}\right]
\end{aligned}
$$




$$
\begin{aligned}
f_{7}\left(x_{t}, x_{H^{+}}\right)= & \frac{1}{2\left(x_{H^{+}}-x_{t}\right)}\left[\frac{\left(x_{t}^{2}+x_{t}-8\right)\left(x_{H^{+}}-x_{t}\right)}{4\left(x_{t}-1\right)^{2}}-\frac{x_{H^{+}}\left(x_{H^{+}}+2\right)}{2\left(x_{H^{+}}-1\right)} \ln x_{H^{+}}\right. \\
& \left.+\frac{x_{H^{+}}\left(x_{t}^{3}-3 x_{t}^{2}+3 x_{t}+2\right)+3\left(x_{t}-2\right) x_{t}^{2}}{2\left(x_{t}-1\right)^{3}} \ln x_{t}\right], \\
f_{8}\left(x_{t}, x_{H^{+}}\right)= & \frac{1}{4\left(x_{H^{+}}-x_{t}\right)}\left[\frac{x_{t} \ln x_{t}}{x_{t}-1}-\frac{x_{H^{+}} \ln x_{H^{+}}}{x_{H^{+}}-1}\right], \\
f_{9}\left(x_{t}, x_{H^{+}}\right)= & \frac{1}{8\left(x_{H^{+}}-x_{t}\right)}\left[\frac{x_{H^{+}}}{x_{H^{+}}-1}+\frac{x_{t}^{2} \ln x_{t}}{\left(x_{t}-1\right)\left(x_{H^{+}}-x_{t}\right)}\right. \\
& \left.-\frac{x_{H^{+}}\left(x_{H^{+}} x_{t}+x_{H^{+}}-2 x_{t}\right)}{\left(x_{H^{+}}-1\right)^{2}\left(x_{H^{+}}-x_{t}\right)} \ln x_{H^{+}}\right], \\
f_{10}\left(x_{t}, x_{H^{+}}\right)= & \frac{1}{8\left(x_{H^{+}}-x_{t}\right)}\left[\frac{x_{H^{+}}-x_{t}}{\left(x_{H^{+}}-1\right)\left(x_{t}-1\right)}+\frac{x_{t}\left(x_{t}-2\right)}{\left(x_{t}-1\right)^{2}} \ln x_{t}-\frac{x_{H^{+}}\left(x_{H^{+}}-2\right)}{\left(x_{H^{+}}-1\right)^{2}} \ln x_{H^{+}}\right] .
\end{aligned}
$$

Open Access. This article is distributed under the terms of the Creative Commons Attribution License (CC-BY 4.0), which permits any use, distribution and reproduction in any medium, provided the original author(s) and source are credited.

\section{References}

[1] ATLAS collaboration, Observation of a new particle in the search for the Standard Model Higgs boson with the ATLAS detector at the LHC, Phys. Lett. B 716 (2012) 1 [arXiv:1207.7214] [INSPIRE].

[2] CMS collaboration, Observation of a new boson at a mass of $125 \mathrm{GeV}$ with the CMS experiment at the LHC, Phys. Lett. B $\mathbf{7 1 6}$ (2012) 30 [arXiv:1207.7235] [INSPIRE].

[3] LHC Higgs Cross Section Working Group collaboration, Handbook of LHC Higgs Cross Sections: 3. Higgs Properties, arXiv:1307.1347 [INSPIRE].

[4] S. Dawson et al., Working Group Report: Higgs Boson, arXiv:1310.8361 [INSPIRE].

[5] A. Pich, The Physics of the Higgs-like Boson, EPJ Web Conf. 60 (2013) 02006 [arXiv: 1307.7700] [INSPIRE].

[6] J. Ellis, Higgs Physics, arXiv:1312.5672 [INSPIRE].

[7] T.D. Lee, A Theory of Spontaneous T Violation, Phys. Rev. D 8 (1973) 1226 [InSPIRE].

[8] G.C. Branco et al., Theory and phenomenology of two-Higgs-doublet models, Phys. Rept. 516 (2012) 1 [arXiv: 1106.0034] [INSPIRE].

[9] J.F. Gunion, H.E. Haber, G.L. Kane and S. Dawson, The Higgs Hunter's Guide, Front. Phys. 80 (2000) 1 [INSPIRE].

[10] S.L. Glashow, J. Iliopoulos and L. Maiani, Weak Interactions with Lepton-Hadron Symmetry, Phys. Rev. D 2 (1970) 1285 [INSPIRE].

[11] S.L. Glashow and S. Weinberg, Natural Conservation Laws for Neutral Currents, Phys. Rev. D 15 (1977) 1958 [INSPIRE]. 
[12] A. Pich and P. Tuzón, Yukawa Alignment in the Two-Higgs-Doublet Model, Phys. Rev. D 80 (2009) 091702 [arXiv:0908.1554] [INSPIRE].

[13] N. Cabibbo, Unitary Symmetry and Leptonic Decays, Phys. Rev. Lett. 10 (1963) 531 [INSPIRE].

[14] M. Kobayashi and T. Maskawa, CP Violation in the Renormalizable Theory of Weak Interaction, Prog. Theor. Phys. 49 (1973) 652 [InSPIRE].

[15] W. Altmannshofer, S. Gori and G.D. Kribs, A Minimal Flavor Violating 2HDM at the LHC, Phys. Rev. D 86 (2012) 115009 [arXiv:1210.2465] [InSPIRE].

[16] Y. Bai, V. Barger, L.L. Everett and G. Shaughnessy, General two Higgs doublet model (2HDM-G) and Large Hadron Collider data, Phys. Rev. D 87 (2013) 115013 [arXiv:1210.4922] [INSPIRE].

[17] V. Barger, L.L. Everett, H.E. Logan and G. Shaughnessy, Scrutinizing h(125) in Two Higgs Doublet Models at the LHC, ILC and Muon Collider, Phys. Rev. D 88 (2013) 115003 [arXiv: 1308.0052] [INSPIRE].

[18] D. López-Val, T. Plehn and M. Rauch, Measuring Extended Higgs Sectors as a Consistent Free Couplings Model, JHEP 10 (2013) 134 [arXiv:1308.1979] [INSPIRE].

[19] L. Wang and X.-F. Han, Status of the aligned two-Higgs-doublet model confronted with the Higgs data, JHEP 04 (2014) 128 [arXiv:1312.4759] [INSPIRE].

[20] A. Celis, V. Ilisie and A. Pich, LHC constraints on two-Higgs doublet models, JHEP 07 (2013) 053 [arXiv: 1302.4022] [INSPIRE].

[21] A. Celis, V. Ilisie and A. Pich, Towards a general analysis of LHC data within two-Higgs-doublet models, JHEP 12 (2013) 095 [arXiv: 1310.7941] [INSPIRE].

[22] M. Jung, A. Pich and P. Tuzón, Charged-Higgs phenomenology in the Aligned two-Higgs-doublet model, JHEP 11 (2010) 003 [arXiv: 1006.0470] [INSPIRE].

[23] M. Jung, A. Pich and P. Tuzón, The B $\rightarrow X s \gamma$ Rate and CP Asymmetry within the Aligned Two-Higgs-Doublet Model, Phys. Rev. D 83 (2011) 074011 [arXiv:1011.5154] [INSPIRE].

[24] M. Jung, X.-Q. Li and A. Pich, Exclusive radiative B-meson decays within the aligned two-Higgs-doublet model, JHEP 10 (2012) 063 [arXiv:1208.1251] [INSPIRE].

[25] A. Celis, M. Jung, X.-Q. Li and A. Pich, Sensitivity to charged scalars in $B \rightarrow D^{(*)} \tau \nu_{\tau}$ and $B \rightarrow \tau \nu_{\tau}$ decays, JHEP 01 (2013) 054 [arXiv: 1210.8443] [INSPIRE].

[26] L. Duarte, G.A. Gonzlez-Sprinberg and J. Vidal, Top quark anomalous tensor couplings in the two-Higgs-doublet models, JHEP 11 (2013) 114 [arXiv:1308.3652] [INSPIRE].

[27] M. Jung and A. Pich, Electric Dipole Moments in Two-Higgs-Doublet Models, JHEP 04 (2014) 076 [arXiv: 1308.6283] [inSPIRE].

[28] H.E. Logan and U. Nierste, $B_{s, d} \rightarrow \ell^{+} \ell^{-}$in a two Higgs doublet model, Nucl. Phys. B 586 (2000) 39 [hep-ph/0004139] [INSPIRE].

[29] C.-S. Huang, W. Liao, Q.-S. Yan and S.-H. Zhu, $B_{s} \rightarrow \ell^{+} \ell^{-}$in a general 2 HDM and MSSM, Phys. Rev. D 63 (2001) 114021 [Erratum ibid. D 64 (2001) 059902] [hep-ph/0006250] [INSPIRE].

[30] C. Bobeth, T. Ewerth, F. Krüger and J. Urban, Analysis of neutral Higgs boson contributions to the decays $\bar{B}_{s} \rightarrow \ell^{+} \ell^{-}$and $\bar{B} \rightarrow K \ell^{+} \ell^{-}$, Phys. Rev. D 64 (2001) 074014 [hep-ph/0104284] [INSPIRE]. 
[31] X.G. He, T.D. Nguyen and R.R. Volkas, B Meson Rare Decays in Two Higgs Doublets Models, Phys. Rev. D 38 (1988) 814 [INSPIRE].

[32] M.J. Savage, Constraining flavor changing neutral currents with $B \rightarrow \mu^{+} \mu^{-}$, Phys. Lett. B 266 (1991) 135 [INSPIRE].

[33] W. Skiba and J. Kalinowski, $B_{s} \rightarrow \tau^{+} \tau^{-}$decay in a two Higgs doublet model, Nucl. Phys. B 404 (1993) 3 [inSPIRE].

[34] E.O. Iltan and G. Turan, $B_{S} \rightarrow \tau^{+} \tau^{-}$decay in the general two Higgs doublet model, JHEP 11 (2002) 031 [hep-ph/0011005] [INSPIRE].

[35] Y.-B. Dai, C.-S. Huang, J.-T. Li and W.-J. Li, CP violation in $B_{d, s} \rightarrow \ell^{+} \ell^{-}$in the model III 2HDM, Phys. Rev. D 67 (2003) 096007 [hep-ph/0301082] [INSPIRE].

[36] P.H. Chankowski and L. Slawianowska, $B_{d, s}^{0} \rightarrow \mu^{-} \mu^{+}$decay in the MSSM, Phys. Rev. D 63 (2001) 054012 [hep-ph/0008046] [INSPIRE].

[37] H. Dreiner, K. Nickel, W. Porod and F. Staub, Full 1-loop calculation of $B R\left(B_{s, d}^{0} \rightarrow \ell \bar{\ell}\right)$ in models beyond the MSSM with SARAH and SPheno, Comput. Phys. Commun. 184 (2013) 2604 [arXiv: 1212.5074] [INSPIRE].

[38] S.R. Choudhury and N. Gaur, Dileptonic decay of B(s) meson in SUSY models with large tan Beta, Phys. Lett. B 451 (1999) 86 [hep-ph/9810307] [inSPIRE].

[39] K.S. Babu and C.F. Kolda, Higgs mediated $B^{0} \rightarrow \mu^{+} \mu^{-}$in minimal supersymmetry, Phys. Rev. Lett. 84 (2000) 228 [hep-ph/9909476] [INSPIRE].

[40] C. Bobeth, A.J. Buras, F. Krüger and J. Urban, $Q C D$ corrections to $B \rightarrow X_{d, s} \nu \bar{\nu}$, $B_{d, s} \rightarrow \ell^{+} \ell^{-}, K \rightarrow \pi \nu \bar{\nu}$ and $K_{L} \rightarrow \mu^{+} \mu^{-}$in the MSSM, Nucl. Phys. B 630 (2002) 87 [hep-ph/0112305] [INSPIRE].

[41] C. Bobeth, T. Ewerth, F. Krüger and J. Urban, Enhancement of $B\left(\bar{B}_{d} \rightarrow \mu^{+} \mu^{-}\right) / B\left(B_{s} \rightarrow \mu^{+} \mu^{-}\right)$in the MSSM with minimal flavor violation and large $\tan \beta$, Phys. Rev. D 66 (2002) 074021 [hep-ph/0204225] [inSPIRE].

[42] A.J. Buras, P.H. Chankowski, J. Rosiek and L. Slawianowska, Correlation between $\Delta M(s)$ and $B_{s, d}^{0} \rightarrow \mu^{+} \mu^{-}$in supersymmetry at large tan beta, Phys. Lett. B 546 (2002) 96 [hep-ph/0207241] [INSPIRE].

[43] A.J. Buras, P.H. Chankowski, J. Rosiek and L. Slawianowska, $\Delta M_{d, s}, B^{0} d, s \rightarrow \mu^{+} \mu^{-}$and $B \rightarrow X_{s} \gamma$ in supersymmetry at large $\tan \beta$, Nucl. Phys. B 659 (2003) 3 [hep-ph/0210145] [INSPIRE].

[44] G. Isidori and A. Retico, $B_{s, d} \rightarrow \ell^{+} \ell^{-}$and $K_{L} \rightarrow \ell^{+} \ell^{-}$in SUSY models with nonminimal sources of flavor mixing, JHEP 09 (2002) 063 [hep-ph/0208159] [INSPIRE].

[45] C.-S. Huang and X.-H. Wu, $B_{s} \rightarrow \mu^{+} \mu^{-}$and $B \rightarrow X(s) \mu^{+} \mu^{-}$in MSSM, Nucl. Phys. B 657 (2003) 304 [hep-ph/0212220] [inSPIRE].

[46] A. Freitas, E. Gasser and U. Haisch, Supersymmetric large $\tan \beta$ corrections to $\Delta M_{d, s}$ and $B_{d, s} \rightarrow \mu^{+} \mu^{-}$revisited, Phys. Rev. D 76 (2007) 014016 [hep-ph/0702267] [INSPIRE].

[47] A. Dedes, J. Rosiek and P. Tanedo, Complete One-Loop MSSM Predictions for B $\rightarrow \ell \ell^{\prime}$ at the Tevatron and LHC, Phys. Rev. D 79 (2009) 055006 [arXiv:0812.4320] [InSPIRE].

[48] A.G. Akeroyd, F. Mahmoudi and D.M. Santos, The decay $B_{s} \rightarrow \mu^{+} \mu^{-}$: updated SUSY constraints and prospects, JHEP 12 (2011) 088 [arXiv:1108.3018] [INSPIRE]. 
[49] A. Arbey, M. Battaglia, F. Mahmoudi and D. Martinez Santos, Supersymmetry confronts $B_{s} \rightarrow \mu^{+} \mu^{-}$: Present and future status, Phys. Rev. D 87 (2013) 035026 [arXiv:1212.4887] [INSPIRE].

[50] J. Albrecht, Brief review of the searches for the rare decays $B_{s}^{0} \rightarrow \mu^{+} \mu^{-}$and $B^{0} \rightarrow \mu^{+} \mu^{-}$, Mod. Phys. Lett. A 27 (2012) 1230028 [arXiv:1207.4287] [INSPIRE].

[51] LHCb collaboration, First Evidence for the Decay $B_{s}^{0} \rightarrow \mu^{+} \mu^{-}$, Phys. Rev. Lett. 110 (2013) 021801 [arXiv: 1211.2674] [INSPIRE].

[52] LHCb collaboration, Measurement of the $B_{s}^{0} \rightarrow \mu^{+} \mu^{-}$branching fraction and search for $B^{0} \rightarrow \mu^{+} \mu^{-}$decays at the LHCb experiment, Phys. Rev. Lett. 111 (2013) 101805 [arXiv: 1307.5024] [INSPIRE].

[53] CMS collaboration, Measurement of the $B_{s} \rightarrow \mu^{+} \mu^{-}$branching fraction and search for $B^{0} \rightarrow \mu^{+} \mu^{-}$with the CMS Experiment, Phys. Rev. Lett. 111 (2013) 101804 [arXiv:1307.5025] [INSPIRE].

[54] CMS and LHCb collaborations, Combination of results on the rare decays $B_{(s)}^{0} \rightarrow \mu^{+} \mu^{-}$ from the CMS and LHCb experiments, CMS-PAS-BPH-13-007 (2013).

[55] C. Bobeth et al., $B_{s, d} \rightarrow \ell^{+} \ell^{-}$in the Standard Model with Reduced Theoretical Uncertainty, Phys. Rev. Lett. 112 (2014) 101801 [arXiv:1311.0903] [INSPIRE].

[56] C. Bobeth, M. Gorbahn and E. Stamou, Electroweak Corrections to $B_{s, d} \rightarrow \ell^{+} \ell^{-}$, Phys. Rev. D 89 (2014) 034023 [arXiv:1311.1348] [INSPIRE].

[57] T. Hermann, M. Misiak and M. Steinhauser, Three-loop QCD corrections to $B_{s} \rightarrow \mu^{+} \mu^{-}$, JHEP 12 (2013) 097 [arXiv: 1311.1347] [INSPIRE].

[58] LHCb collaboration, Implications of LHCb measurements and future prospects, Eur. Phys. J. C 73 (2013) 2373 [arXiv:1208.3355] [InSPIRE].

[59] S. Davidson and H.E. Haber, Basis-independent methods for the two-Higgs-doublet model, Phys. Rev. D 72 (2005) 035004 [Erratum ibid. D 72 (2005) 099902] [hep-ph/0504050] [INSPIRE].

[60] H.E. Haber and D. O'Neil, Basis-independent methods for the two-Higgs-doublet model. II. The Significance of tan beta, Phys. Rev. D 74 (2006) 015018 [hep-ph/0602242] [INSPIRE].

[61] H.E. Haber and D. O'Neil, Basis-independent methods for the two-Higgs-doublet model III: The CP-conserving limit, custodial symmetry and the oblique parameters $S, T, U$, Phys. Rev. D 83 (2011) 055017 [arXiv: 1011.6188] [INSPIRE].

[62] D. O'Neil, Phenomenology of the Basis-Independent CP-Violating Two-Higgs Doublet Model [Dissertation], arXiv:0908.1363 [INSPIRE].

[63] H. Serodio, Yukawa Alignment in a Multi Higgs Doublet Model: An effective approach, Phys. Lett. B 700 (2011) 133 [arXiv:1104.2545] [INSPIRE].

[64] I. de Medeiros Varzielas, Family symmetries and alignment in multi-Higgs doublet models, Phys. Lett. B 701 (2011) 597 [arXiv:1104.2601] [INSPIRE].

[65] G. Cree and H.E. Logan, Yukawa alignment from natural flavor conservation, Phys. Rev. D 84 (2011) 055021 [arXiv:1106.4039] [INSPIRE].

[66] A. Pich, Flavour constraints on multi-Higgs-doublet models: Yukawa alignment, Nucl. Phys. Proc. Suppl. 209 (2010) 182 [arXiv:1010.5217] [INSPIRE]. 
[67] G. Cvetič, C.S. Kim and S.S. Hwang, Higgs mediated flavor changing neutral currents in the general framework with two Higgs doublets: An RGE analysis, Phys. Rev. D 58 (1998) 116003 [hep-ph/9806282] [INSPIRE].

[68] P.M. Ferreira, L. Lavoura and J.P. Silva, Renormalization-group constraints on Yukawa alignment in multi-Higgs-doublet models, Phys. Lett. B 688 (2010) 341 [arXiv:1001.2561] [INSPIRE].

[69] C.B. Braeuninger, A. Ibarra and C. Simonetto, Radiatively induced flavour violation in the general two-Higgs doublet model with Yukawa alignment, Phys. Lett. B 692 (2010) 189 [arXiv: 1005.5706] [INSPIRE].

[70] J. Bijnens, J. Lu and J. Rathsman, Constraining General Two Higgs Doublet Models by the Evolution of Yukawa Couplings, JHEP 05 (2012) 118 [arXiv:1111.5760] [INSPIRE].

[71] A.J. Buras, R. Fleischer, J. Girrbach and R. Knegjens, Probing New Physics with the $B_{s}$ to $\mu^{+} \mu^{-}$Time-Dependent Rate, JHEP 07 (2013) 077 [arXiv:1303.3820] [INSPIRE].

[72] K. De Bruyn et al., Probing New Physics via the $B_{s}^{0} \rightarrow \mu^{+} \mu^{-}$Effective Lifetime, Phys. Rev. Lett. 109 (2012) 041801 [arXiv: 1204.1737] [INSPIRE].

[73] R. Fleischer, On Branching Ratios of $B_{s}$ Decays and the Search for New Physics in $B_{s}^{0} \rightarrow \mu^{+} \mu^{-}$, Nucl. Phys. Proc. Suppl. 241-242 (2013) 135 [arXiv:1208.2843] [INSPIRE].

[74] W. Altmannshofer, P. Paradisi and D.M. Straub, Model-Independent Constraints on New Physics in $b \rightarrow s$ Transitions, JHEP 04 (2012) 008 [arXiv:1111.1257] [INSPIRE].

[75] G. Buchalla, A.J. Buras and M.E. Lautenbacher, Weak decays beyond leading logarithms, Rev. Mod. Phys. 68 (1996) 1125 [hep-ph/9512380] [INSPIRE].

[76] A.J. Buras, Weak Hamiltonian, CP-violation and rare decays, hep-ph/9806471 [INSPIRE].

[77] T. Hahn, Generating Feynman diagrams and amplitudes with FeynArts 3, Comput. Phys. Commun. 140 (2001) 418 [hep-ph/0012260] [INSPIRE].

[78] N.D. Christensen and C. Duhr, FeynRules - Feynman rules made easy, Comput. Phys. Commun. 180 (2009) 1614 [arXiv:0806.4194] [InSPIRE].

[79] A. Alloul, J. D'Hondt, K. De Causmaecker, B. Fuks and M. Rausch de Traubenberg, Automated mass spectrum generation for new physics, Eur. Phys. J. C 73 (2013) 2325 [arXiv: 1301.5932] [INSPIRE].

[80] A. Alloul, N.D. Christensen, C. Degrande, C. Duhr and B. Fuks, FeynRules $2.0-a$ complete toolbox for tree-level phenomenology, arXiv:1310.1921 [INSPIRE].

[81] V.A. Smirnov, Asymptotic expansions in momenta and masses and calculation of Feynman diagrams, Mod. Phys. Lett. A 10 (1995) 1485 [hep-th/9412063] [InSPIRE].

[82] V.A. Smirnov, Applied asymptotic expansions in momenta and masses, Springer Tracts Mod. Phys. 177 (2002) 1 [INSPIRE].

[83] C. Bobeth, M. Misiak and J. Urban, Photonic penguins at two loops and $m_{t}$ dependence of $B R\left[B \rightarrow X_{s} \ell^{+} \ell^{-}\right]$, Nucl. Phys. B 574 (2000) 291 [hep-ph/9910220] [INSPIRE].

[84] M.E. Peskin and D.V. Schroeder, An Introduction to quantum field theory, Addison-Wesley, Reading U.S.A. (1995), pg. 842.

[85] G. Buchalla, A.J. Buras and M.K. Harlander, Penguin box expansion: Flavor changing neutral current processes and a heavy top quark, Nucl. Phys. B 349 (1991) 1 [INSPIRE]. 
[86] F.J. Botella and C.S. Lim, Flavor Changing Yukawa Coupling in the Standard Model and Muon Polarization in $K_{L} \rightarrow \mu \bar{\mu}$, Phys. Rev. Lett. 56 (1986) 1651 [INSPIRE].

[87] T. Inami and C.S. Lim, Effects of Superheavy Quarks and Leptons in Low-Energy Weak Processes $K_{L} \rightarrow \mu \bar{\mu}, K^{+} \rightarrow \pi^{+} \nu \bar{\nu}$ and $K^{0} \leftrightarrow \bar{K}^{0}$, Prog. Theor. Phys. 65 (1981) 297 [Erratum ibid. 65 (1981) 1772] [INSPIRE].

[88] G. Buchalla and A.J. Buras, The rare decays $K \rightarrow \pi \nu \bar{\nu}, B \rightarrow X \nu \bar{\nu}$ and $B \rightarrow \ell^{+} \ell: A n$ Update, Nucl. Phys. B 548 (1999) 309 [hep-ph/9901288] [InSPIRE].

[89] G. Buchalla and A.J. Buras, $Q C D$ corrections to rare $K$ and $B$ decays for arbitrary top quark mass, Nucl. Phys. B 400 (1993) 225 [INSPIRE].

[90] G. Buchalla and A.J. Buras, QCD corrections to the $\bar{s} d Z$ vertex for arbitrary top quark mass, Nucl. Phys. B 398 (1993) 285 [inSPIRE].

[91] M. Misiak and J. Urban, QCD corrections to FCNC decays mediated by $Z$ penguins and $W$ boxes, Phys. Lett. B 451 (1999) 161 [hep-ph/9901278] [INSPIRE].

[92] B. Grzadkowski and P. Krawczyk, Higgs Particle effects in flavor changing transitions, Z. Phys. C 18 (1983) 43 [inSPIRE].

[93] P. Krawczyk, Flavor Changing Yukawa Coupling of the Standard Higgs Boson: Effects of the External Quark Masses, Z. Phys. C 44 (1989) 509 [INSPIRE].

[94] Particle Data Group collaboration, J. Beringer et al., Review of Particle Physics (RPP), Phys. Rev. D 86 (2012) 010001 [INSPIRE], and 2013 partial update for the 2014 edition.

[95] ATLAS, CDF, CMS and D0 collaborations, First combination of Tevatron and LHC measurements of the top-quark mass, arXiv:1403.4427 [INSPIRE].

[96] S. Aoki et al., Review of lattice results concerning low energy particle physics, arXiv:1310.8555 [INSPIRE]. Updates at http://itpwiki.unibe.ch/flag.

[97] Heavy Flavor Averaging Group collaboration, Y. Amhis et al., Averages of B-Hadron, C-Hadron and tau-lepton properties as of early 2012, arXiv:1207.1158 [INSPIRE].

[98] P. Gambino and C. Schwanda, Inclusive semileptonic fits, heavy quark masses and $V_{c b}$, Phys. Rev. D 89 (2014) 014022 [arXiv:1307.4551] [INSPIRE].

[99] CKMfitTer Group collaboration, J. Charles et al., CP violation and the CKM matrix: Assessing the impact of the asymmetric B factories, Eur. Phys. J. C 41 (2005) 1 [hep-ph/0406184] [INSPIRE]. Updated results and plots available at: http://ckmfitter.in2p3.fr.

[100] M. Ciuchini et al., 2000 CKM triangle analysis: a critical review with updated experimental inputs and theoretical parameters, JHEP 07 (2001) 013 [hep-ph/0012308] [INSPIRE]. Updated results and plots available at: http://www.utfit.org.

[101] M. Awramik, M. Czakon, A. Freitas and G. Weiglein, Precise prediction for the $W$ boson mass in the standard model, Phys. Rev. D 69 (2004) 053006 [hep-ph/0311148] [INSPIRE].

[102] K.G. Chetyrkin, J.H. Kuhn and M. Steinhauser, RunDec: A Mathematica package for running and decoupling of the strong coupling and quark masses, Comput. Phys. Commun. 133 (2000) 43 [hep-ph/0004189] [INSPIRE].

[103] C. McNeile, C.T.H. Davies, E. Follana, K. Hornbostel and G.P. Lepage, High-Precision $f_{B_{s}}$ and HQET from Relativistic Lattice QCD, Phys. Rev. D 85 (2012) 031503 [arXiv:1110.4510] [INSPIRE]. 
[104] Fermilab Lattice and MILC collaborations, A. Bazavov et al., B- and D-meson decay constants from three-flavor lattice QCD, Phys. Rev. D 85 (2012) 114506 [arXiv:1112.3051] [INSPIRE].

[105] N.H. Christ et al., B-meson decay constants from 2+1-flavor lattice QCD with domain-wall light quarks and relativistic heavy quarks, arXiv:1404.4670 [INSPIRE].

[106] HPQCD collaboration, R.J. Dowdall, C.T.H. Davies, R.R. Horgan, C.J. Monahan and J. Shigemitsu, B-Meson Decay Constants from Improved Lattice Nonrelativistic QCD with Physical u, d, s and c Quarks, Phys. Rev. Lett. 110 (2013) 222003 [arXiv:1302.2644] [INSPIRE].

[107] N. Carrasco et al., $A N_{f}=2+1+1$ "twisted" determination of the b-quark mass, $f_{B}$ and $f_{B_{s}}$, arXiv: 1311.2837 [INSPIRE].

[108] A. Lenz and U. Nierste, Numerical Updates of Lifetimes and Mixing Parameters of B Mesons, arXiv:1102.4274 [INSPIRE]. 